NBER WORKING PAPER SERIES

\title{
ESTIMATION OF DYNAMIC DISCRETE CHOICE MODELS IN CONTINUOUS TIME WITH AN APPLICATION TO RETAIL COMPETITION
}

\author{
Peter Arcidiacono \\ Patrick Bayer \\ Jason R. Blevins \\ Paul B. Ellickson \\ Working Paper 18449 \\ http://www.nber.org/papers/w18449 \\ NATIONAL BUREAU OF ECONOMIC RESEARCH \\ 1050 Massachusetts Avenue \\ Cambridge, MA 02138 \\ October 2012
}

We thank the attendees of the 2009 Cowles Foundation Conference on Structural Microeconomics, the 2010 cemmap Conference on Matching and Sorting, and the 2012 NBER/NSF/CEME Conference on the Econometrics of Dynamic Games as well as seminar participants at Chicago (Economics and Booth), Duke, Harvard, Johns Hopkins, Kentucky, London School of Economics, Michigan, Ohio State (Economics and Fisher), Penn State, Rochester, Toronto, UBC, UC Davis, UCLA, Virginia, Western Ontario, Washington University (Olin), Wisconsin, and Yale for useful comments. Timothy Schwuchow provided excellent research assistance. The views expressed herein are those of the authors and do not necessarily reflect the views of the National Bureau of Economic Research.

NBER working papers are circulated for discussion and comment purposes. They have not been peerreviewed or been subject to the review by the NBER Board of Directors that accompanies official NBER publications.

(C) 2012 by Peter Arcidiacono, Patrick Bayer, Jason R. Blevins, and Paul B. Ellickson. All rights reserved. Short sections of text, not to exceed two paragraphs, may be quoted without explicit permission provided that full credit, including $(\mathcal{C}$ notice, is given to the source. 
Estimation of Dynamic Discrete Choice Models in Continuous Time with an Application

to Retail Competition

Peter Arcidiacono, Patrick Bayer, Jason R. Blevins, and Paul B. Ellickson

NBER Working Paper No. 18449

October 2012, Revised Fabruary 2015

JEL No. C13,C35,L11,L13

\begin{abstract}
This paper develops a dynamic model of retail competition and uses it to study the impact of the expansion of a new national competitor on the structure of urban markets. In order to accommodate substantial heterogeneity (both observed and unobserved) across agents and markets, the paper first develops a general framework for estimating and solving dynamic discrete choice models in continuous time that is computationally light and readily applicable to dynamic games. In the proposed framework, players face a standard dynamic discrete choice problem at decision times that occur stochastically. The resulting stochastic-sequential structure naturally admits the use of CCP methods for estimation and makes it possible to compute counterfactual simulations for relatively high-dimensional games. The model and method are applied to the retail grocery industry, into which Wal-Mart began rapidly expanding in the early 1990s, eventually attaining a dominant position. We find that Wal-Mart's expansion into groceries came mostly at the expense of the large incumbent supermarket chains, rather than the single-store outlets that bore the brunt of its earlier conquest of the broader general merchandise sector. Instead, we find that independent grocers actually thrive when Wal-Mart enters, leading to an overall reduction in market concentration. These competitive effects are strongest in larger markets and those into which Wal-Mart expanded most rapidly, suggesting a diminishing role of scale and a greater emphasis on differentiation in this previously mature industry.
\end{abstract}

Peter Arcidiacono

Department of Economics

201A Social Sciences Building

Duke University

Durham, NC 27708

and NBER

psarcidi@econ.duke.edu

Patrick Bayer

Department of Economics

Duke University

213 Social Sciences

Durham, NC 27708

and NBER

patrick.bayer@duke.edu
Jason R. Blevins

Department of Economics

Ohio State University

Columbus, $\mathrm{OH}$

blevins.141@osu.edu

Paul B. Ellickson

Simon School of Business Administration

University of Rochester

Rochester, NY 14627

Email: paul.ellickson@simon.rochester.edu

paul.ellickson@simon.rochester.edu 


\section{Introduction}

Beginning with the static equilibrium entry models of Bresnahan and Reiss (1991) and Berry (1992), the modern empirical literature in industrial organization has sought to understand the determinants of market structure and its impact on market power and the nature of competition in oligopolistic industries. Recent papers have extended these models to permit forward-looking behavior on the part of firms, as well as more complex forms of investment and post-entry competition. ${ }^{1}$ Adding such dynamic considerations broadens the nature of strategic interactions among firms and permits the study of a range of fundamentally dynamic phenomena such as preemption, predation, and limit pricing.

Using existing methods, incorporating forward-looking behavior in models of strategic interaction has been computationally costly, making it infeasible to compute the dynamic equilibrium unless the state space is sufficiently small. As a result, empirical researchers have often had to sacrifice much of the rich firm and market heterogeneity that can be incorporated in static models in order to study the kinds of interesting strategic behavior that can result when firms are forward-looking. With this trade-off in mind, the central goal of this paper is to provide a new approach for computing dynamic equilibrium models of market competition that is much lighter computationally and, therefore, permits the study of dynamic behavior without preventing researchers from incorporating dimensions of heterogeneity (both observed and unobserved) that may be critical for understanding key aspects of the nature of market competition.

At the outset, it is important to clarify that the key computational challenge in the dynamic games literature is related to the computation of the dynamic equilibrium (e.g., for use in counterfactuals) rather than estimation per se. In particular, since the seminal work of Hotz and Miller (1993) and Hotz, Miller, Sanders, and Smith (1994), conditional choice probability (CCP) estimators have been applied to a wide range of dynamic discrete choice problems including, more recently, simultaneous-move dynamic games (Aguirregabiria and Mira, 2007, Bajari et al., 2007, Pesendorfer and Schmidt-Dengler, 2007, Pakes et al., 2007). The key advantage of CCP estimation is that it eliminates the need to compute the full solution of the dynamic game, allowing empirical researchers to estimate relatively highdimensional games, including many that can not be solved directly even once.

While knowledge of the key parameters of many dynamic games can be informative, a key limitation of the application of CCP estimation to high-dimensional problems is that it

\footnotetext{
${ }^{1}$ Building on earlier methodological contributions pioneered by Aguirregabiria and Mira (2007), Bajari, Benkard, and Levin (2007), Pesendorfer and Schmidt-Dengler (2007), and Pakes, Ostrovsky, and Berry (2007), empirical researchers have recently examined the impact of environmental regulations on entry, investment and market power in the cement industry (Ryan, 2012), the effect of demand fluctuations in the concrete industry (Collard-Wexler, 2013), and the impact of increased royalty fees on the variety of products offered by commercial radio stations (Sweeting, 2013).
} 
is often impossible to compute counterfactual simulations of the estimated model. In the context of games, the calculation of players' expectations over all combinations of actions of their rivals grows exponentially in the number of players, making it computationally challenging to compute the equilibrium even in some relatively simple economic contexts. ${ }^{2}$

In this paper, we develop a characterization of a dynamic game in continuous time that not only alleviates some of the computational difficulties associated with simultaneous move games, but also links naturally with the existing literature on dynamic discrete choice models and dynamic discrete games. The key feature of our approach is that players face a standard dynamic discrete choice problem at decision times that occur stochastically. The resulting stochastic-sequential structure naturally admits the use of CCP methods for estimation and makes it possible to compute counterfactual simulations for relatively high-dimensional games.

CCP estimation applied to our formulation of a dynamic game in continuous time has several important advantages that carry over from the discrete time literature. Most directly, CCP estimation continues to eliminate the need to compute the full solution of the model for estimation. Using our framework, the two-step estimators of Aguirregabiria and Mira (2002, 2007), Bajari et al. (2007), Hotz et al. (1994), Pakes et al. (2007), and Pesendorfer and Schmidt-Dengler (2007) can be applied in continuous time. In most empirical studies, the equilibrium will only need to be computed a handful of times to perform the counterfactual analyses conducted in the paper. In addition, it is straightforward to account for unobserved heterogeneity with our framework by extending the methods of Arcidiacono and Miller (2011). We demonstrate both of these advantages in our empirical application, applying the methods to a high dimensional problem while incorporating unobserved heterogeneity, an important feature of the institutional setting.

But, importantly, CCP estimation has additional advantages in continuous time beyond those studied in the discrete time literature. In particular, it is easier to satisfy the finite dependence property of Arcidiacono and Miller $(2011,2014)$ and Altuğ and Miller (1998), whereby only a handful of conditional choice probabilities are needed to express the future utility term. This occurs because the inversion theorem of Hotz and Miller (1993) yields a mapping between differences in value functions and conditional choice probabilities in the continuous time setting, as opposed to differences in conditional value functions and conditional choice probabilities in the discrete time setting. As we illustrate in Section 3, working with value functions rather than conditional value functions also has the added benefit of limiting the need to estimate conditional choice probabilities for rare events, a prevalent feature in many empirical settings.

\footnotetext{
${ }^{2}$ These limitations have led some to suggest alternatives to the Markov perfect equilibrium concept in which firms condition on long run averages (regarding rivals' states) instead of current information (Weintraub, Benkard, and Van Roy, 2008).
} 
We take advantage of this new formulation of a dynamic game in continuous time to study the impact of a new national competitor on the structure of urban markets across the United States. Specifically, we examine the impact of Wal-Mart's rapid expansion into the retail grocery industry from $1994-2006 .{ }^{3}$ In particular, we model the decisions of Wal-Mart and its rivals over whether to operate grocery stores in a market and at what scale (i.e., number of stores). We include the choices of Wal-Mart and up to seven competing chains, as well as the single-store entry and exit decisions of several dozen fringe players. Each geographic market is characterized by observed features - most importantly, the level and growth rate of population - as well as unobserved heterogeneity that affects the relative profitability of Wal-Mart, chain, and fringe stores in that market.

This characterization of the problem results in a dynamic game that has a rich error structure (due to the unobserved heterogeneity) and an enormous number of states. We estimate the model using CCP methods and solve counterfactually for the equilibrium under several scenarios designed to measure how Wal-Mart's entry into the retail grocery industry affects the profitability and decision-making of rival chain and fringe firms. ${ }^{4}$

The estimates imply that Wal-Mart's entry has a substantial impact on market structure that is heterogeneous both across markets and firm types. In particular, we find that Wal-Mart's expansion came mostly at the expense of the large, incumbent grocery chains, leading some to exit the market completely and sharply diminishing the scale of others. In contrast, the small fringe firms actually thrive in the new market structure, suggesting a greater ability to differentiate themselves (e.g., in terms of product offerings or geography) from both Wal-Mart and the remaining chains. Taken as a whole, market concentration is sharply reduced by Wal-Mart's entry in these markets.

Notably, this new, entrepreneurial activity is strongest in the larger markets and those into which Wal-Mart expanded the fastest. In contrast, in another set of (primarily smaller, Western) markets, Wal-Mart's entry greatly concentrates the market in a way that closely resembles the impact of its initial entry into discount retailing two decades earlier (Jia, 2008). Wal-Mart's entry is felt most directly by the fringe firms in these markets because, much like the rural markets Wal-Mart focused on originally, chain stores were less established in these markets to begin with. However, in the vast majority of grocery markets, Wal-Mart actually benefits the fringe.

A comparison of the results for specifications with and without unobserved heterogeneity reveals that the inclusion of unobserved heterogeneity is essential for uncovering these qual-

\footnotetext{
${ }^{3}$ Our methods have also been applied by Nevskaya and Albuquerque (2012) to online games, by Schiraldi, Smith, and Takahasi (2012) to supermarkets, by Mazur (2014) to airlines, and by Cosman (2014) to bars in Chicago.

${ }^{4}$ Our counterfactual policy simulations involve calculating value functions at up to 157 million states in each of 205 markets, yet by taking advantage of the continuous time formulation this is computationally feasible.
} 
itatively distinct economic implications of Wal-Mart's entry across markets. While there is still some variation across markets, the specification without unobserved heterogeneity implies that Wal-Mart's entry decreases market concentration in every market in the sample and sharply understates the positive impact on small, independent stores, especially in the larger markets. Taken as a whole, the results of our analysis demonstrate the importance of incorporating substantial heterogeneity both across markets and firm types in estimating dynamic games of retail entry and competition, thereby highlighting the advantage of computationally light approaches for estimating and solving dynamic models with large state spaces.

Our paper relates to the literature on both estimation of dynamic models in continuous time as well as the empirical literature on entry in retail markets. ${ }^{5}$ Continuous-time methods have a long history in the search literature. Our approach is most connected to the literature in which job offers arrive at an exogenous rate. Another vein of the search literature, however, assumes that individuals receive offers according to their search intensity, effectively choosing a hazard rate for job offers. This is the spirit of Doraszelski and Judd (2012) who first illustrated the computational advantages of casting dynamic games into continuous time. Players in their model make simultaneous, continuous decisions that control the hazard rate of state changes (e.g., choose an investment hazard which results stochastically in a discrete productivity gain). In our paper, we take advantage of the insight of Doraszelski and Judd (2012) regarding how continuous time, and more specifically sequential state-to-state transitions, can reduce the computational burden of dynamic games but reframe the problem in a way that naturally parallels the discrete time discrete choice literature and retains the computational advantages of CCP methods in estimation as well as circumventing issues of multiple equilibria in estimation.

The paper is structured as follows. Section 2 introduces our model in a simple singleagent context in order to build intuition. Section 3 develops an alternative CCP representation of the value function which will facilitate two-step estimation of the model. Section 4 extends the model to the multi-agent setting. Concrete and canonical examples are provided in both the single- and multi-agent cases. Section 5 develops our estimators and discusses issues associated with time aggregation. Section 6 introduces and describes the results of our empirical analysis of the market structure of grocery store chains in geographically separate U.S. markets. Section 7 concludes.

\footnotetext{
${ }^{5}$ On the empirical side, our paper is the first to estimate structurally the impact of Wal-Mart on both chain and a single store firms. Ellickson and Grieco (2013) examine the impact of Wal-Mart on the structure of the supermarket industry using descriptive methods from the treatment effects literature, while Basker and Noel (2009) and Matsa (2011) look at its impact on prices and quality. Wal-Mart's previous experience in the discount industry has been analyzed by Jia (2008), Holmes (2011), and Ellickson, Houghton, and Timmins (2010).
} 


\section{Single-Agent Dynamic Discrete Choice Models}

In this section, we introduce a dynamic discrete choice model of single-agent decisionmaking in continuous time. The single-agent problem provides a simple setting in which to describe the main features of our continuous time framework. We show how these extend directly to multi-agent settings in the following section. We begin this section by laying out the notation and structure of the model in a general context. We then introduce an example - the classic bus engine (capital) replacement model of Rust (1987) — to illustrate how to apply our model in a familiar setting.

Consider a dynamic single-agent decision problem in which time is continuous, indexed by $t \in[0, \infty)$. The state of the model at any time $t$ can be summarized by an element $k$ of some finite state space $\mathcal{X}=\{1, \ldots, K\}$. Two competing Poisson processes drive the dynamics of the model. First, a finite-state Markov jump process on $\mathcal{X}$ with a $K \times K$ intensity matrix $Q_{0}$ governs moves by nature exogenous state changes that are not a result of actions by the agent. The elements of $Q_{0}$ are the rates at which particular state transitions occur. Second, a Poisson arrival process with rate parameter $\lambda$ governs when the agent can move. When a move opportunity arrives, the agent chooses an action $j$ from among $J$ alternatives in a discrete choice set $\mathcal{A}=\{0, \ldots, J-1\}$, conditional on the current state $k$.

Before describing the agent's problem, we review some properties of finite Markov jump processes, which are the basic building blocks of our model and can be used to characterize both exogenous and endogenous state changes on $\mathcal{X}$. A finite Markov jump process on $\mathcal{X}$ is a stochastic process $X_{t}$ indexed by $t \in[0, \infty)$. At any time $t$, the process remains at $X_{t}$ for a random time interval $\tau$ (the holding time) before transitioning to some new state $X_{t+\tau}$. A sample path of such a process is a piecewise-constant, right-continuous function of time. Jumps occur according to a Poisson process and the holding times between jumps are exponentially distributed.

A finite-state Markov jump process can be characterized by an intensity matrix, which contains the rate parameters for each possible state transition:

$$
Q=\left[\begin{array}{cccc}
q_{11} & q_{12} & \ldots & q_{1 K} \\
q_{21} & q_{22} & \ldots & q_{2 K} \\
\vdots & \vdots & \ddots & \vdots \\
q_{K 1} & q_{K 2} & \ldots & q_{K K}
\end{array}\right]
$$

For $l \neq k$

$$
q_{k l}=\lim _{h \rightarrow 0} \frac{\operatorname{Pr}\left(X_{t+h}=l \mid X_{t}=k\right)}{h}
$$


is the hazard rate for transitions from state $k$ to state $l$ and

$$
q_{k k}=-\sum_{l \neq k} q_{k l}
$$

is the overall rate at which the process leaves state $k$ (and hence, $q_{k k}$ is negative). Transitions out of state $k$ follow an exponential distribution with rate parameter $-q_{k k}$ and, conditional on leaving state $k$, the process transitions to $l \neq k$ with probability $q_{k l} / \sum_{l^{\prime} \neq k} q_{k l^{\prime}}$. For additional details about Markov jump processes see, for example, Karlin and Taylor (1975, Section 4.8).

Returning to the agent's problem, we assume that the agent is forward-looking and discounts future payoffs at rate $\rho$. Exogenous state changes that the agent cannot control occur according to a Markov jump process with intensity matrix $Q_{0}$. While in state $k$, the agent receives flow utility $u_{k}$. At rate $\lambda \in(0, \infty)$ the agent makes a decision, choosing an action $j \in \mathcal{A}$ and receiving an instantaneous payoff $\psi_{j k}+\varepsilon_{j}$, where $\psi_{j k}$ is the mean payoff (or cost) associated with making choice $j$ in state $k$ and $\varepsilon_{j} \in \mathbb{R}$ is an instantaneous choicespecific payoff shock. ${ }^{6}$ Let $\sigma_{j k}$ denote the probability that the agent optimally chooses choice $j$ in state $k$. The agent's choice may result in a deterministic state change. Let $l(j, k)$ denote the state that results upon making choice $j$ in state $k$.

We can now derive the instantaneous Bellman equation, a recursive expression for the value function $V_{k}$ which gives the present discounted value of all future payoffs obtained from starting in some state $k$ and behaving optimally in future periods. For small time increments $h$, under the Poisson assumption, the probability of an event with rate $\lambda$ occurring is $\lambda h$. Given the discount rate $\rho$, the discount factor for such increments is $1 /(1+\rho h)$. Thus, for small time increments $h$ the optimal discounted value in state $k$ satisfies

$$
\begin{aligned}
V_{k}=\frac{1}{1+\rho h}\left[u_{k} h+\sum_{l \neq k} q_{k l} h V_{l}+\lambda h \operatorname{Exax}_{j}\left\{\psi_{j k}+\varepsilon_{j}\right.\right. & \left.+V_{l(j, k)}\right\} \\
& \left.+\left(1-\lambda h-\sum_{l \neq k} q_{k l} h\right) V_{k}+o(h)\right] .
\end{aligned}
$$

Rearranging and letting $h \rightarrow 0$, we obtain the following recursive expression for $V_{k}$ :

$$
V_{k}=\frac{u_{k}+\sum_{l \neq k} q_{k l} V_{l}+\lambda \operatorname{Emax}_{j}\left\{\psi_{j k}+\varepsilon_{j}+V_{l(j, k)}\right\}}{\rho+\lambda+\sum_{l \neq k} q_{k l}} .
$$

\footnotetext{
${ }^{6}$ Although the choice-specific shocks $\varepsilon_{j}$ vary over time, we omit the $t$ subscript for notational simplicity. For convenience, we also assume the distribution of $\varepsilon_{j}$ is independent of the state, to avoid conditioning on $k$ throughout, but the joint distribution of the vector $\varepsilon=\left(\varepsilon_{0}, \ldots, \varepsilon_{J-1}\right)^{\top}$ can, in general, depend on $k$ without additional difficulties.
} 
The denominator contains the sum of the discount factor and the rates of all possible state changes. The numerator is composed of the flow payoff for being in state $k$, the rate-weighted values associated with exogenous state changes, and the expected current and future value obtained when a move arrival occurs in state $k$. The expectation is with respect to the joint distribution of $\varepsilon=\left(\varepsilon_{0}, \ldots, \varepsilon_{J-1}\right)^{\top}$. Alternatively, and perhaps more intuitively, rearranging once again shows that the instantaneous discounted increment to the value function $V_{k}$ is

$$
\rho V_{k}=u_{k}+\sum_{l \neq k} q_{k l}\left(V_{l}-V_{k}\right)+\lambda \operatorname{E} \max _{j}\left\{\psi_{j k}+\varepsilon_{j}+V_{l(j, k)}-V_{k}\right\}
$$

A policy rule is a function $\delta: \mathcal{X} \times \mathbb{R}^{J} \rightarrow \mathcal{A}$ which assigns to each state $k$ and vector $\varepsilon$ an action from $\mathcal{A}$. The optimal policy rule satisfies the following inequality condition, where $V_{k}$ is the value function that solves the Bellman equation: ${ }^{7}$

$$
\delta(k, \varepsilon)=j \Longleftrightarrow \psi_{j k}+\varepsilon_{j}+V_{l(j, k)} \geq \psi_{j^{\prime} k}+\varepsilon_{j^{\prime}}+V_{l\left(j^{\prime}, k\right)} \quad \forall j^{\prime} \in \mathcal{A} .
$$

That is, when given the opportunity to choose an action, $\delta$ assigns the action that maximizes the agent's expected future discounted payoff. Thus, under the optimal policy rule, the conditional choice probabilities are

$$
\sigma_{j k}=\operatorname{Pr}[\delta(k, \varepsilon)=j \mid k]
$$

Note that the move arrival rate, $\lambda$, and the choice probabilities of the agent, $\sigma_{j k}$, also imply a Markov jump process on $\mathcal{X}$ with intensity matrix $Q_{1}$, where $Q_{1}$ is a function of both $\lambda$ and $\sigma_{j k}$ for all $j$ and $k$. In particular, the hazard rate of action $j$ in state $k$ is simply $\lambda \sigma_{j k}$, the product of the move arrival rate and the choice probability. The choice probability $\sigma_{j k}$ is thus the proportion of moves in state $k$, which occur at rate $\lambda$, that result in action $j$. Summing the individual intensity matrices yields the aggregate intensity matrix $Q=Q_{0}+Q_{1}$ of the compound process, which fully characterizes the state transition dynamics of the model. This simple and intuitive structure is especially important in extending the model to include multiple agents, and in accomodating estimation with discrete time data. We discuss both of these extensions in subsequent sections.

\subsection{Example: A Single-Agent Renewal Model}

Our first example is a simple single-agent renewal model, based on the bus engine replacement problem analyzed by Rust (1987). The single state variable captures the accumulated

\footnotetext{
${ }^{7}$ Note that in estimation we would substitute in for the value function using equation (2), as we will be interested in recovering the flow payoffs.
} 
mileage of a bus engine. Let $q_{k 1}$ and $q_{k 2}$ denote the rates at which one- and two-unit mileage increments occur, respectively. With each move arrival, the agent faces a binary choice: replace the engine $(j=1)$ or continue $(j=0)$. If the agent replaces the engine, the mileage is reset to the initial state $k=1$ and the agent pays a replacement $\operatorname{cost} c$. The agent faces a cost minimization problem where the flow cost incurred in mileage state $k$ is represented by $u_{k}$. The value function for mileage state $k$ is

$$
V_{k}=\frac{u_{k}+q_{k 1} V_{k+1}+q_{k 2} V_{k+2}+\lambda \operatorname{Emax}\left\{V_{k}+\varepsilon_{0}, V_{0}+c+\varepsilon_{1}\right\}}{\rho+q_{k 1}+q_{k 2}+\lambda},
$$

where, in our general notation from before, the instantaneous payoffs are

$$
\psi_{j k}= \begin{cases}0, & \text { if } j=0 \\ c, & \text { if } j=1\end{cases}
$$

We will return to this example in the following section, where we discuss a useful CCP representation of the value function.

\section{CCP Representation}

The primary difference between our framework and traditional discrete time dynamic discrete choice models is that, rather that having state changes and choices made simultaneously at pre-determined intervals, only one event occurs at any given instant (almost surely), with random time intervals between moves. Given that the unobserved preferences evolve stochastically as in the discrete time literature, we are able to show that some of the insights of Hotz and Miller (1993), Altuğ and Miller (1998), and Arcidiacono and Miller (2011, 2014) on expressing value functions in terms of conditional choice probabilities (CCPs) apply here as well. In fact, as we show below, it is actually much easier to express the value functions in terms of CCPs in the continuous time setting than in discrete time. In this way we can often eliminate the value functions on the right-hand side of (2), implying that no fixed point problem needs to be solved in estimation.

Let $\sigma_{k}=\left(\sigma_{0 k}, \ldots, \sigma_{J-1, k}\right)^{\top}$ denote the vector of CCPs in state $k$. We make the following standard assumptions on the discount rate and the choice-specific shocks (Rust, 1994).

Assumption 1. $\rho>0$.

Assumption 2. The choice-specific shocks $\varepsilon$ are iid over time and across choices with a known joint distribution which is absolutely continuous with respect to Lebesgue measure, has finite first moments, and support equal to $\mathbb{R}^{J}$. 
With these two assumptions, it is possible to express the continuous-time value function as a function of the flow and instantaneous payoffs, conditional choice probabilities, $\sigma$, and hazards for nature, $Q_{0}$, analogous to Aguirregabiria and Mira (2002):

Proposition 1. If Assumptions 1 and 2 hold, then the value function can be written as

$$
V(\sigma)=\left[(\rho+\lambda) I-\lambda \Sigma(\sigma)-Q_{0}\right]^{-1}[u+\lambda E(\sigma)]
$$

where $I$ is the $K \times K$ identity matrix, $\Sigma(\sigma)$ is the $K \times K$ state transition matrix induced by the choice probabilities $\sigma$ due to actions by the agent, $u$ is the $K \times 1$ vector of flow payoffs, and $E(\sigma)$ is the $K \times 1$ vector containing the ex-ante expected values of the instantaneous payoffs in each state, $\sum_{j} \sigma_{j k}\left[\psi_{j k}+e_{j k}\left(\sigma_{k}\right)\right]$ where $e_{j k}(\sigma)$ is the expected value of $\varepsilon_{j k}$ given that choice $j$ is optimal,

$$
\frac{1}{\sigma_{j k}} \int \varepsilon_{j k} \cdot 1\left\{\varepsilon_{j^{\prime} k}-\varepsilon_{j k} \leq \psi_{j k}-\psi_{j^{\prime} k}+V_{l(j, k)}(\sigma)-V_{l\left(j^{\prime}, k\right)}(\sigma) \forall j^{\prime}\right\} f\left(\varepsilon_{k}\right) d \varepsilon_{k} .
$$

Proof. See Appendix B.

This formulation provides the basis for some of our estimators. However, this representation still requires an inverse of a potentially large (though sparse) matrix. In the remainder of this section we develop conditions under which an inversion is not needed and the value function can be expressed as a function of the conditional choice probabilities for a limited number of states.

To do so, we first derive two results that allow us to link value functions across states. The first is essentially the continuous-time analog of Proposition 1 of Hotz and Miller (1993). Namely, using the conditional choice probabilities, we can derive relationships between the value functions associated with any two states as long as both states are feasible from the initial state, should the agent have the right to move.

Proposition 2. If Assumptions 1 and 2 hold, then there exists a function $\Gamma^{1}\left(j, j^{\prime}, \sigma_{k}\right)$ such that for all $j, j^{\prime} \in \mathcal{A}$,

$$
V_{l(j, k)}=V_{l\left(j^{\prime}, k\right)}+\psi_{j^{\prime} k}-\psi_{j k}+\Gamma^{1}\left(j, j^{\prime}, \sigma_{k}\right)
$$

Proof. See Appendix B.

Thus, the value functions themselves can be linked across states. This is similar to the result of Proposition 1 of Hotz and Miller (1993), which applied to conditional value functions: the value of making a particular choice conditional on behaving optimally in the future. 
The second result establishes a similar CCP representation for the final term in the Bellman equation.

Proposition 3. If Assumptions 1 and 2 hold, then there exists a function $\Gamma^{2}\left(j^{\prime}, \sigma_{k}\right)$ such that for all $j^{\prime} \in \mathcal{A}$,

$$
\operatorname{E} \max _{j}\left\{\psi_{j k}+\varepsilon_{j}+V_{l(j, k)}\right\}=V_{l\left(j^{\prime}, k\right)}+\psi_{j^{\prime} k}+\Gamma^{2}\left(j^{\prime}, \sigma_{k}\right)
$$

Proof. See Appendix B.

The intuition for Proposition 3 is that we can express the left hand side of (5) relative to $V_{l\left(j^{\prime}, k\right)}+\psi_{j^{\prime} k}$ for an action $j^{\prime}$ of our choosing. By doing so, the terms inside the E max term will consist of differences in value functions and instantaneous payoffs. These differences, as established by Proposition 2, can be expressed as functions of conditional choice probabilities.

For a concrete example of these two propositions, consider the case where the $\varepsilon$ 's follow the type I extreme value distribution. In this case, closed form expressions exist for both $\Gamma^{2}$ and $\Gamma^{1}$ :

$$
\begin{aligned}
\Gamma^{1}\left(j, j^{\prime}, \sigma_{k}\right) & =\ln \left(\sigma_{j k}\right)-\ln \left(\sigma_{j^{\prime} k}\right) \\
\Gamma^{2}\left(j^{\prime}, \sigma_{k}\right) & =-\ln \left(\sigma_{j^{\prime} k}\right)+\gamma
\end{aligned}
$$

where $\gamma$ is Euler's constant.

Importantly, Proposition 2 allows us to link value functions across many states. For example, suppose that action 0 is a continuation action which does not change the state, $l(0, k)=k$, and has no instantaneous payoff or cost, $\psi_{0 k}=0$. If in state $k$ the agent is able to move to $k^{\prime}$ by taking action $j^{\prime}$, and is further able to move from $k^{\prime}$ to $k^{\prime \prime}$ by taking action $j^{\prime \prime}$, then it is possible to express $V_{k^{\prime \prime}}$ as a function of $V_{k}$ by substituting in the relevant relationships:

$$
\begin{aligned}
V_{k} & =V_{k^{\prime}}+\psi_{j^{\prime}, k}+\Gamma^{1}\left(0, j^{\prime}, \sigma_{k}\right) \\
& =V_{k^{\prime \prime}}+\psi_{j^{\prime \prime}, k^{\prime}}+\psi_{j^{\prime}, k}+\Gamma^{1}\left(0, j^{\prime \prime}, \sigma_{k^{\prime}}\right)+\Gamma^{1}\left(0, j^{\prime}, \sigma_{k}\right) .
\end{aligned}
$$

By successively linking value functions to other value functions, in many cases it is straightforward to find a chain such that the remaining value functions on the right hand side of (2) can be expressed in terms of $V_{k}$ and conditional choice probabilities. Then, collecting all terms involving $V_{k}$ yields an expression for $V_{k}$ in terms of the flow payoff of state $k$ and the conditional choice probabilities. Since the latter can often be flexibly estimated directly from the data and the former is an economic primitive, it is no longer necessary to solve 
a dynamic programming problem to obtain the value functions. This is formalized in the following result.

Definition. A state $k^{*}$ is attainable from state $k$ if there exists a sequence of actions from $k$ that result in state $k^{*}$.

Proposition 4. Suppose that Assumptions 1 and 2 hold and that for a given state $k, j=0$ is a continuation action with $l(0, k)=k$ and for all states $l \neq k$ with $q_{k l}>0$ there exists a state $k^{*}$ that is attainable from both $k$ and $l$. Then, there exists a function $\Gamma_{k}\left(\psi, Q_{0}, \lambda, \sigma\right)$ such that

$$
\rho V_{k}=u_{k}+\Gamma_{k}\left(\psi, Q_{0}, \lambda, \sigma\right)
$$

Proof. See Appendix B.

The function $\Gamma_{k}$ for each state may depend on the model primitives $\psi, Q_{0}$, and $\lambda$ as well as the CCPs, $\sigma$. By restating the problem in this way, when the conditional choice probabilities are available, no fixed point problem needs to be solved in order to obtain the value functions and no $K \times K$ matrix need be stored or inverted. This can often lead to large computational gains. We now provide an example of how to apply these propositions.

\subsection{Example: A Single-Agent Renewal Model}

Recall the bus engine replacement example of Section 2.1, where the value function was characterized by (3). Applying Proposition 3 eliminates the third term in the numerator:

$$
V_{k}=\frac{u_{k}+q_{k 1} V_{k+1}+q_{k 2} V_{k+2}+\lambda \Gamma^{2}\left(0, \sigma_{k}\right)}{\rho+q_{k 1}+q_{k 2}} .
$$

Although there is no direct link between the value function at $k$ and the value functions at $k+1$ and $k+2$, it is possible to link the two value functions through the replacement decision. In particular, $V_{k}$ and $V_{k+1}$ can be expressed as follows:

$$
\begin{aligned}
V_{k} & =V_{0}+c+\Gamma^{1}\left(0,1, \sigma_{k}\right), \\
V_{k+1} & =V_{0}+c+\Gamma^{1}\left(0,1, \sigma_{k+1}\right) .
\end{aligned}
$$

This implies that we can express $V_{k+1}$ in terms of $V_{k}$ :

$$
V_{k+1}=V_{k}+\Gamma^{1}\left(0,1, \sigma_{k+1}\right)-\Gamma^{1}\left(0,1, \sigma_{k}\right)
$$


Using a similar expression for $V_{k+2}$, we obtain the function $\Gamma_{k}$ from Proposition 4:

$$
\begin{aligned}
\Gamma_{k}\left(\psi, Q_{0}, \lambda, \sigma\right)=q_{k 1} \Gamma^{1}\left(0,1, \sigma_{k+1}\right)+q_{k 2} \Gamma^{1}\left(0,1, \sigma_{k+2}\right) & \\
& -\left(q_{k 1}+q_{k 2}\right) \Gamma^{1}\left(0,1, \sigma_{k}\right)+\lambda \Gamma^{2}\left(0, \sigma_{k}\right) .
\end{aligned}
$$

This example illustrates one of the benefits of continuous time over discrete time when using conditional choice probabilities. Namely, as illustrated by Arcidiacono and Miller (2011), forming renewal problems using CCPs required both expressing the future utility term relative to a particular choice and differencing the conditional valuation functions used in estimation. In this example, the future utility terms for both replacing and continuing would be expressed relative to the value of replacing. Hence accurate estimates of the conditional probability of replacing at very low mileages would be needed, but these are low probability events whose estimates will likely depend heavily on the smoothing parameters or functional forms used to mitigate the associated small sample problems. In the present continuous time framework, differencing is not required in order to form the value function in terms of conditional choice probabilities and we only need the replacement probabilities at states $k, k+1$, and $k+2$.

\section{Dynamic Discrete Games}

The potential advantages of modeling decisions using a continuous time framework are particularly salient in games, where the state space is often enormous. Working in continuous time highlights aspects of strategic interaction that are muted by discrete time (e.g., firstmover advantage) and mitigates unnatural implications that can arise from simultaneity (e.g., ex post regret). In fact, a number of recent papers in the empirical games literature (e.g., Einav, 2010, Schmidt-Dengler, 2006) have adopted a sequential structure for decisionmaking to accommodate the underlying economic theory associated with their games.

Extending the single-agent model of Section 2 to the case of dynamic discrete games with many players is simply a matter of modifying the intensity matrix governing the state transitions to incorporate players' beliefs regarding the future actions of their rivals. We begin this section by describing the structure of the model, followed by properties of equilibrium strategies and beliefs. We then show how to apply the CCP representation results of Section 3 in the context of dynamic games.

Suppose there are $N$ players indexed by $i=1, \ldots, N$. As before, the state space $\mathcal{X}$ is finite with $K$ elements. This is without loss of generality, since each of these elements may be regarded as indices of elements in a higher-dimensional, but finite, space of firmmarket-specific state vectors. Player $i$ 's choice set in state $k$ is $\mathcal{A}_{i k}$. For simplicity, we 
consider the case where each player has $J$ actions in all states: $\mathcal{A}_{i k}=\{0, \ldots, J-1\}$ for all $i$ and $k$. We index the remaining model primitives by $i$, including the flow payoffs in state $k, u_{i k}$, instantaneous payoffs, $\psi_{i j k}$, and choice probabilities, $\sigma_{i j k}$. Let $l(i, j, k)$ denote the continuation state that arises after player $i$ makes choice $j$ in state $k$. We assume that players share a common discount rate $\rho$.

Although it is still sufficient to have only a single jump process on $\mathcal{X}$, with some intensity matrix $Q_{0}$, to capture moves by nature, there are now $N$ independent, competing Poisson processes with rate $\lambda \in(0, \infty)$ generating move arrivals for each of the $N$ players. ${ }^{8}$ The next event to occur is determined by the earliest arrival of one of these $N+1$ processes.

Let $\varsigma_{i}$ denote player $i$ 's beliefs regarding the actions of rival players, given by a collection of $(N-1) \times J \times K$ probabilities $\varsigma_{i m j k}$ for each rival player $m \neq i$, state $k$, and choice $j$. Applying Bellman's principal of optimality (Bellman, 1957), the value function for an active player $i$ in state $k$ can be defined recursively as ${ }^{9}$

$V_{i k}\left(\varsigma_{i}\right)=\frac{u_{i k}+\sum_{l \neq k} q_{k l} V_{i l}\left(\varsigma_{i}\right)+\sum_{m \neq i} \lambda \sum_{j} \varsigma_{i m j k} V_{i, l(m, j, k)}\left(\varsigma_{i}\right)+\lambda \operatorname{Emax}_{j}\left\{\psi_{i j k}+\varepsilon_{i j}+V_{i, l(i, j, k)}\left(\varsigma_{i}\right)\right\}}{\rho+\sum_{l \neq k} q_{k l}+N \lambda}$.

The denominator of this expression the sum of the discount factor and the rates of exogenous state changes and moves by players. The numerator can be understood by looking at each term separately. The first term is the flow payoff that accrues to firm $i$ each instant the model remains in state $k$. Next, we have a sum over possible exogenous state changes, weighted by the rates at which those changes occur. The third term is a sum over rival firms, in particular, over the rate-weighted state changes that could result due to the action of a rival firm. The final term is the rate-weighted continuation value that occurs when the agent moves and optimally makes a discrete choice.

Following Maskin and Tirole (2001), we focus on Markov perfect equilibria in pure strategies, as is standard in the discrete-time games literature. A Markov strategy for player $i$ is a mapping which assigns an action from $\mathcal{A}_{i k}$ to each state $\left(k, \varepsilon_{i}\right) \in \mathcal{X} \times \mathbb{R}^{J}$. Focusing on Markov strategies eliminates the need to condition on the full history of play.

Given beliefs for each player, $\left\{\varsigma_{1}, \ldots, \varsigma_{N}\right\}$, and a collection of model primitives, a Markov strategy for player $i$ is a best response if ${ }^{10}$

$$
\delta_{i}\left(k, \varepsilon_{i} ; \varsigma_{i}\right)=j \Longleftrightarrow \psi_{i j k}+\varepsilon_{i j}+V_{i, l(i, j, k)}\left(\varsigma_{i}\right) \geq \psi_{i j^{\prime} k}+\varepsilon_{i j^{\prime}}+V_{i, l\left(i, j^{\prime}, k\right)}\left(\varsigma_{i}\right) \quad \forall j^{\prime} \in \mathcal{A}_{i k} .
$$

Then, given the distribution of choice-specific shocks, each Markov strategy $\delta_{i}$ implies re-

\footnotetext{
${ }^{8}$ For simplicity, we assume the move arrival rates are equal for each firm.

${ }^{9}$ If a player is not active in state $k$, then the probability of inaction $(j=0)$ for that player is set to one.

${ }^{10}$ In the event of a tie, we assume the action with the smallest index is assigned. Because the distribution of $\varepsilon_{i}$ is continuous under Assumption 2, such ties occur with probability zero.
} 
sponse probabilities for each choice in each state:

$$
\sigma_{i j k}=\operatorname{Pr}\left[\delta_{i}\left(k, \varepsilon_{i} ; \varsigma_{i}\right)=j \mid k\right]
$$

Definition. A collection of Markov strategies $\left\{\delta_{1}, \ldots, \delta_{N}\right\}$ and beliefs $\left\{\varsigma_{1}, \ldots, \varsigma_{N}\right\}$ is a Markov perfect equilibrium if for all $i$ :

1. $\delta_{i}\left(k, \varepsilon_{i}\right)$ is a best response given beliefs $\varsigma_{i}$, for all $k$ and almost every $\varepsilon_{i}$;

2. for all players $m \neq i$, the beliefs $\varsigma_{m i}$ are consistent with the best response probabilities implied by $\delta_{i}$, for each $j$ and $k$.

Following Milgrom and Weber (1985) and Aguirregabiria and Mira (2007), we can characterize Markov perfect equilibria in probability space, rather than in terms of pure Markov strategies, as a collection of equilibrium best response probabilities $\left\{\sigma_{1}, \ldots, \sigma_{N}\right\}$ where each probability in $\sigma_{i}$ is a best response given beliefs $\sigma_{-i}$. Likewise, any such collection of probabilities can be extended to a Markov perfect equilibrium.

In particular, equilibrium conditional choice probabilities are fixed points to the best

response probability mapping, which defines a continuous function from $[0,1]^{N \times J \times K}$ onto itself. Existence of an equilibrium then follows from Brouwer's Theorem, as established by the following proposition.

Proposition 5. A Markov perfect equilibrium exists.

Proof. See Appendix B.

\subsection{CCP Representation}

As in single-agent models, the value function for any player $i$ in the multi-player case can be expressed in terms of reduced-form CCPs and hazards. The following proposition formalizes this and generalizes Proposition 1 to the multi-player setting.

Proposition 6. If Assumptions 1 and 2 hold, then the value function for each player $i$ can be written as

$$
V_{i}(\sigma)=\left[(\rho+N \lambda) I-\sum_{m=1}^{N} \lambda \Sigma_{m}\left(\sigma_{m}\right)-Q_{0}\right]^{-1}\left[u_{i}+\lambda E_{i}\left(\sigma_{i}\right)\right]
$$

where $\Sigma_{m}\left(\sigma_{m}\right)$ is the $K \times K$ state transition matrix induced by the actions of player $m$ given the choice probabilities $\sigma_{m}$ and where $E_{i}\left(\sigma_{i}\right)$ is a $K \times 1$ vector where each element $k$ is the ex-ante expected value of the choice-specific payoff in state $k, \sum_{j} \sigma_{i j k}\left[\psi_{i j k}+e_{i j k}\left(\sigma_{i k}\right)\right]$ 
where $e_{i j k}(\sigma)$ is the expected value of $\varepsilon_{i j k}$ given that choice $j$ is optimal,

$$
\frac{1}{\sigma_{i j k}} \int \varepsilon_{i j k} \cdot 1\left\{\varepsilon_{i j^{\prime} k}-\varepsilon_{i j k} \leq \psi_{i j k}-\psi_{i j^{\prime} k}+V_{i, l(i, j, k)}(\sigma)-V_{i, l\left(i, j^{\prime}, k\right)}(\sigma) \forall j^{\prime}\right\} f\left(\varepsilon_{i k}\right) d \varepsilon_{i k} .
$$

Proof. See Appendix B.

We also note that the propositions in Section 3 apply to games as well in cases where the attainability condition holds. Hence, it is possible to eliminate the value functions in the fourth term of the numerator of (7) using Proposition 3:

$$
V_{i k}=\frac{u_{i k}+\sum_{l \neq k} q_{k l} V_{i l}+\sum_{m \neq i} \lambda \sum_{j} \sigma_{m j k} V_{i, l(m, j, k)}+\lambda \Gamma_{i}^{2}\left(0, \sigma_{i k}\right)}{\rho+\sum_{l \neq k} q_{k l}+N \lambda} .
$$

Eliminating the other value functions, however, is problematic as each player may only be able to move the process to some subset of the state space via a unilateral action, since they only have direct control over their own state. For example, when firms have different numbers of stores, a given firm is only able to choose its own stores, not the stores of its competitors. There are at least two cases where the remaining value functions can be eliminated. The first is the case where there is a terminal choice, such as permanently exiting a market. Since no further choices are made by the focal firm, the value function for the terminal choice does not include other value functions. A concrete example is provided below. The other case is where an action can be taken to reset the system for all players. For example, consider a game that involves technology adoption. By achieving a particular technology level, previous technologies may become obsolete, effectively renewing the states of the other players.

\subsection{Example: Multi-Store Entry and Exit}

Note that in either the terminal or reset case, there only has to be an attainable scenario where the agent can execute the terminal or reset action. For example, consider a game amongst retailers where firms compete by opening and closing stores. Given a move arrival, a firm can build a store, $j=1$, do nothing, $j=0$, or, if the agent has at least one store, close a store, $j=-1$. Once a firm has no stores, it makes no further choices. Let $c$ denote the scrap value of closing a store.

Suppose that firm $i$ has $k_{i}$ stores and the economy-wide state is $k=\left(k_{1}, \ldots, k_{N}\right)$, the store counts of all firms in the market (including potential entrants, namely firms with zero stores). Let $l^{*}\left(i, k, k_{i}^{\prime}\right)$ denote the state that is equal to the initial state $k$, but where firm $i$ has $k_{i}^{\prime}$ stores instead of $k_{i}$. Applying Proposition 2 and normalizing the value of zero stores 
to zero, we can express $V_{i k}$ as:

$$
V_{i k}=\sum_{k_{i}^{\prime}=1}^{k_{i}} \Gamma^{1}\left(0,-1, \sigma_{i, l^{*}\left(i, k, k_{i}^{\prime}\right)}\right)+k_{i} c .
$$

Since (11) holds for all $k$, we can use the value of fully exiting to link value functions for any pair of states. Namely, linking the value functions on the right hand side of (10) to $V_{i k}$ and solving for $V_{i k}$ yields:

$$
\begin{aligned}
\rho V_{i k} & =u_{i k}+\lambda_{i} \Gamma_{i}^{2}\left(0, \sigma_{i k}\right) \\
& +\sum_{m \neq i} \sigma_{m,-1, k}\left(\sum_{k_{i}^{\prime}=1}^{k_{i}}\left[\Gamma_{i}^{1}\left(0,-1, \sigma_{i, l^{*}\left(i, l(m,-1, k), k_{i}^{\prime}\right)}\right)-\Gamma_{i}^{1}\left(0,-1, \sigma_{i, l^{*}\left(i, k, k_{i}^{\prime}\right)}\right)\right]\right) \\
& +\sum_{m \neq i} \sigma_{m, 1, k}\left(\sum_{k_{i}^{\prime}=1}^{k_{i}}\left[\Gamma_{i}^{1}\left(0,-1, \sigma_{i, l^{*}\left(i, l(m, 1, k), k_{i}^{\prime}\right)}\right)-\Gamma_{i}^{1}\left(0,-1, \sigma_{i, l^{*}\left(i, k, k_{i}^{\prime}\right)}\right)\right]\right) .
\end{aligned}
$$

Once again, no fixed point calculation is required to express the full value function, a simplification that is especially powerful in the context of high-dimensional discrete games.

\section{Estimation}

We now turn to estimation. Methods that solve for the value function directly and use it to obtain the implied choice probabilities for estimation are referred to as full-solution methods. The nested fixed point (NFXP) algorithm of Rust (1987), which uses value function iteration inside of an optimization routine that maximizes the likelihood, is the classic example of a full-solution method.

CCP-based estimation methods, on the other hand, are two-step methods pioneered by Hotz and Miller (1993) and Hotz et al. (1994) and later extended by Aguirregabiria and Mira (2002, 2007), Bajari et al. (2007), Pesendorfer and Schmidt-Dengler (2007), Pakes et al. (2007), and Arcidiacono and Miller (2011). The CCPs are estimated in a first step and used to approximate the value function in a closed-form inversion or simulation step. The approximate value function is then used in the likelihood function or the GMM criterion function to estimate the structural parameters.

Full-solution methods have the advantage that the full structure of the model is imposed in estimation. However, these methods can become quite computationally expensive for complex models with many players or a large state space. Many candidate parameter vectors must be evaluated during estimation and, if the value function is costly to compute, even if solving the model once might be feasible, doing so many times may not be. In 
the presence of multiple equilibria, they also require researchers to make an assumption on the equilibrium selection mechanism and solve for all the equilibria to complete the model (Bajari, Hong, and Ryan, 2007). In addition to allowing the value function to be computed very quickly, CCP methods provide an attractive solution to the issue of multiplicity. Under the usual assumption invoked in the literature (e.g., Aguirregabiria and Mira, 2007), that conditional on observables, all observations are generated by the same equilibrium, then the resulting likelihood conditions on the equilibrium that is played in the data, bypassing the need to consider other equilibria. We formalize this assumption below.

Our model has the advantage of being estimable via either the full solution or CCP approaches when applicable. As in Doraszelski and Judd (2012), the use of continuous time reduces the computational burden of computing expectations over successor states because only a single state change can occur at any particular instant. An attractive and novel feature of our framework is that it is also easily estimable using standard CCP methods. This greatly reduces the computational costs of estimation relative to full-solution methods. Having estimated a large problem with CCP methods, it is then straightforward to use the same model for post-estimation exercises, since the computational burden of computing the equilibrium a few times for these purposes is not as great as nesting several such solutions into an estimation routine. In this way, our framework preserves a tight link between the estimated model and that used for post-estimation analysis, something which has proven infeasible for many empirical applications that have been modeled in discrete time.

In the rest of this section we describe the estimation algorithms. We begin with the simplest case in which continuous-time data is available and we consider both the fullsolution and two-step estimators in this case. Note that the full-solution estimator is only applicable to single agent models or in cases where there is a unique equilibrium to the strategic game, but we consider it here primarily to build intuition for the estimators that follow. Next, since data is often reported only at discrete intervals, we next show how our methods can be applied to discrete time data. We then extend the methods to incorporate permanent unobserved heterogeneity. Finally, we discuss nonparametric identification of the model.

Throughout, we normalize $\lambda$, the arrival rate of moves for players, to one, implying an average of one move per unit of time (e.g., one year). In a discrete time model, this is similar to the assumption that players move once per time period, but it only restricts the average not the actual realized move times. In other words, it allows players to move more or less than once per unit of time by allowing randomness in the realized move times rather than restricting moves to occur exactly at unit intervals. 


\subsection{Continuous Time Data}

\subsubsection{Full-Solution Estimation with Continuous Time Data}

We maintain the convention that choice $j=0$ for each agent is a continuation choice which does not change the state. Then, in state $k$, the probability of the next state change occurring during an interval of length $\tau$ is

$$
1-\exp \left[-\tau\left(\sum_{l \neq k} q_{k l}+\sum_{i} \lambda \sum_{j \neq 0} \sigma_{i j k}\right)\right] \text {. }
$$

This is the cdf of the exponential distribution with rate parameter equal to the sum of the exogenous state transition rates and the hazards of the non-continuation actions for each player.

Differentiating with respect to $\tau$ yields the density for the time of the next state change, which is the exponential pdf with the same rate parameter as before:

$$
\left(\sum_{l \neq k} q_{k l}+\sum_{i} \lambda \sum_{j \neq 0} \sigma_{i j k}\right) \exp \left[-\tau\left(\sum_{l \neq k} q_{k l}+\sum_{i} \lambda \sum_{j \neq 0} \sigma_{i j k}\right)\right] .
$$

Conditional on a state change occurring in state $k$, the probability that the change is due to agent $i$ taking action $j$ is

$$
\frac{\lambda \sigma_{i j k}}{\sum_{l \neq k} q_{k l}+\sum_{i} \lambda \sum_{j \neq 0} \sigma_{i j k}}
$$

Now, define the function

$$
g(\tau, k ; q, \theta)=\exp \left[-\tau\left(\sum_{l \neq k} q_{k l}+\sum_{i} \lambda \sum_{j \neq 0} \sigma_{i j k}\right)\right],
$$

which is the second term from (12), noting that the vector of equilibrium choice probabilities $\sigma=\left(\sigma_{i j k}\right)$ is, implicitly, a function of $q=\operatorname{vec}\left(Q_{0}\right)$ and the parameters $\theta$.

Then, the joint likelihood of the next stage change occurring after an interval of length $\tau$ and being the result of player $i$ taking action $j$ is the product of (13) and (14),

$$
\lambda \sigma_{i j k} g(\tau, k ; q, \theta)
$$

with the corresponding likelihood of nature moving the state from $k$ to $l$ being

$$
q_{k l} g(\tau, k ; q, \theta)
$$


Now consider a sample of $M$ markets where in each market $m$ we observe a sequence $\left\{k_{m n}\right\}_{n=1}^{T}$ of $T$ state changes occurring over the interval $[0, \bar{T}]$. Let $k_{m n}$ denote the state prior to the $n$-th state change in market $m$, with $k_{m, T+1}$ denoting the final state. Let $t_{m n}$ denote the time of the $n$-th event and let $\tau_{m n}$ denote the holding time between events, defined as $\tau_{m n}=t_{m n}-t_{m, n-1}$ for $n \leq T$. For the interval between the last event and the end of the sampling period, we define $\tau_{m, T+1}=\bar{T}-t_{m T}$. Let $I_{m n}(i, j)$ be the indicator for whether the $n$-th move in market $m$ was a move by player $i$ and the choice was $j$ and let $I_{m n}(0, l)$ be the indicator for whether the $n$-th move in market $m$ was a move by nature to state $l$. The maximum likelihood estimates of $q$ and $\theta$ are then the solution to:

$$
\begin{aligned}
(\hat{q}, \hat{\theta})=\arg \max _{(q, \theta)}\left\{\sum_{m=1}^{M}\right. & \sum_{n=1}^{T}\left[\ln g\left(\tau_{m n}, k_{m n} ; q, \theta\right)+\sum_{l \neq k_{m n}} I_{m n}(0, l) \ln q_{k l}\right. \\
& \left.\left.\quad+\sum_{i} \sum_{j \neq 0} I_{m n}(i, j) \ln \left(\lambda \sigma_{i j k}(q, \theta)\right)\right]+\ln g\left(\tau_{m, N+1}, k_{m, N+1} ; q, \theta\right)\right\} .
\end{aligned}
$$

Note that embedded in the estimation problem is the solution to a fixed point problem which is needed in order to obtain the value functions and CCPs. We have made the dependence of the choice probabilities on the parameters and rates explicit. The last term in the expression is the natural logarithm of one minus the exponential cdf, to account for the fact that another state change was not observed by the end of the sampling period. Note that $(\hat{q}, \hat{\theta})$ is a full-information maximum likelihood estimator and is $\sqrt{M}$-consistent, asymptotically normal, and efficient under standard regularity assumptions.

\subsubsection{Two-Step Estimation with Continuous Time Data}

As discussed in Section 3, it is possible to express differences in continuous time value functions as functions of the conditional choice probabilities. These expressions can sometimes be used in such a way that solving the nested fixed point problem is unnecessary. In this section, we show how two-step methods apply in estimation, linking reduced form hazards to conditional choice probabilities.

Step 1: Estimating the Reduced-Form Hazards Let $h_{i j k}=\lambda \sigma_{i j k}$ denote the hazard for an active player $i$ choosing action $j$ in state $k$ and let

$$
h=\left(q_{12}, q_{13}, \ldots, q_{K-1, K}, \lambda \sigma_{111}, \ldots, \lambda \sigma_{1 J k}, \ldots \lambda \sigma_{N 11}, \ldots, \lambda \sigma_{N J K}\right)
$$

denote the vector of distinct hazards for nature and all state-specific, non-continuation action hazards of players and let $\mathcal{H} \subset \mathbb{R}^{K(K-1)+N J K}$ denote the space of all such vectors $h$. 
In Step 1, one estimates the hazards $h_{i j k}$ nonparametrically. For example, these hazards can be estimated by maximum likelihood by writing the exponential cdf in (12) as a function of $h_{i j k}$ instead of $\lambda \sigma_{i j k}$. Similarly, we can rewrite the function in (15) as

$$
\tilde{g}(\tau, k ; h)=\exp \left[-\tau\left(\sum_{l \neq k} q_{k l}+\sum_{i} \sum_{j \neq 0} h_{i j k}\right)\right] .
$$

Then, the maximum likelihood estimates of the hazards $h$ are

$$
\begin{aligned}
\tilde{h}=\arg \max _{h \in \mathcal{H}}\left\{\sum _ { m = 1 } ^ { M } \sum _ { n = 1 } ^ { T } \left[\operatorname { l n } \tilde { g } \left(\tau_{m n},\right.\right.\right. & \left.k_{m n} ; h\right)+\sum_{l \neq k_{m n}} I_{m n}(0, l) \ln q_{k l} \\
& \left.\left.+\sum_{i} \sum_{j \neq 0} I_{m n}(i, j) \ln h_{i j k}\right]+\ln \tilde{g}\left(\tau_{m, N+1}, k_{m, N+1} ; h\right)\right\} .
\end{aligned}
$$

Step 2: Estimating the Structural Payoff Parameters In Step 2, we use the estimated reduced-form hazards to estimate the structural payoff parameters $\theta$. Given the estimated hazards and a value of $\lambda$, we can estimate the conditional choice probabilities for $j \neq 0$ as $\tilde{h}_{i j k} / \lambda$ and $1-\sum_{j \neq 0} \tilde{h}_{i j k} / \lambda$ for $j=0$.

We can then express the structural conditional choice-specific hazards as functions of $\theta$ and the estimated hazards $\tilde{h}$ so that no fixed-point problem needs to be solved. When the finite dependence condition holds, this simply involves evaluating the mapping in (6). Otherwise, we can use the matrix representation in $(9)$. Let $\breve{h}_{i j k}(\tilde{h}, \theta)$ denote the particular mapping used. Similarly, let $\check{g}(\tau, k ; \tilde{h}, \theta)$ denote the mapping in $(15)$ with $\check{h}_{i j k}(\tilde{h}, \theta)$ used in place of $\lambda \sigma_{i j k}$.

Since we already have estimates of the rates $q$ (which are the first $K(K-1)$ components of $\tilde{h}$ ), we focus on estimating $\theta$. The joint likelihood of the next state change occurring after an interval of length $\tau$ and being the result of player $i$ taking action $j$ is

$$
\check{h}_{i j k}(\tilde{h}, \theta) \check{g}(\tau, k ; \tilde{h}, \theta) \text {. }
$$

The second stage estimates are then

$$
\begin{aligned}
\check{\theta}=\arg \max _{\theta}\left\{\sum_{m=1}^{M} \sum_{n=1}^{T}\left[\ln \check{g}\left(\tau_{m n}, k_{m n} ; \tilde{h}, \theta\right)+\sum_{i} \sum_{j \neq 0} I_{m n}(i, j) \ln \check{h}_{i j k}(\tilde{h}, \theta)\right]\right. \\
\left.+\ln \check{g}\left(\tau_{m, N+1}, k_{m, N+1} ; \tilde{h}, \theta\right)\right\} .
\end{aligned}
$$

This is a fairly standard two-step estimator and is consistent and asymptotically normal 
under suitable regularity conditions In the next section we consider two-step estimation in the leading case of discrete time data. There, we formally state sufficient conditions for consistency and asymptotic normality. The properties of the estimator above, with continuous time data, are largely similar.

\subsection{Discrete Time Data}

Often the exact sequence of events and event times is not observed, rather, the state is only observed at discrete points in time. Here, we consider estimation with discretely-sampled data. First we consider full-solution estimation and then we show that one can easily carry out two-step estimation in an analogous manner. We proceed by assuming the data are sampled from independent markets at uniformly spaced time intervals of length $\Delta .^{11}$

Assumption 3. The observations $\left\{k_{m n}: m=1, \ldots, M, n=1, \ldots, T\right\}$, sampled at times on the lattice $\{n \Delta: n=1, \ldots, T\}$, are independent across markets $m$ and $\operatorname{Pr}\left(k_{m n}=k\right)>0$ for all $k \in \mathcal{X}$.

Let $P_{k l}(\Delta)$ denote the probability that the system has transitioned to state $l$ after a period of length $\Delta$ given that it was initially in state $k$, given the aggregate intensity matrix $Q$. The corresponding matrix of these probabilities, $P(\Delta)=\left(P_{k l}(\Delta)\right)$, is the transition matrix, which satisfies

$$
P(\Delta)=\mathrm{e}^{\Delta Q}=\sum_{j=0}^{\infty} \frac{(\Delta Q)^{j}}{j !} .
$$

This quantity is the matrix exponential, the matrix analog of the scalar exponential, which can be computed using one of many known algorithms (cf. Moler and Loan, 1978, Sidje, 1998). ${ }^{12}$

The transition probabilities summarize the relevant information about a pair of observations $k_{m, n-1}$ and $k_{m n}$. That is, $P_{k_{m, n-1}, k_{m n}}(\Delta)$ is the probability of the process moving from $k_{m, n-1}$ to $k_{m n}$ after an interval of length $\Delta$. This includes cases where $k_{m n}=k_{m, n-1}$, since the transition probabilities account for the case of no jumps at all, as well as all sequences involving any number of jumps to intermediate states before returning to the initial state.

\subsubsection{Computational Considerations}

Since the $Q$ matrix can be large, this may seem to introduce a dimensionality problem rivaling that of discrete time models. However, the $Q$ matrix is often very sparse, which

\footnotetext{
${ }^{11}$ Extending this to cases with irregular time intervals is straightforward and also helps with identification. See Cuthbert (1973), Singer and Spilerman (1976), Hansen and Sargent (1983), and Blevins (2013) for results on identification of continuous time models with irregularly spaced observations.

${ }^{12}$ The matrix exponential operator is available, for example, in Matlab, via the expm function.
} 
substantially reduces the computational burden. Sparse matrix algorithms can be used to compute $P(\Delta)$ which typically require only being able to compute the action of $Q$ on some generic vector $v$. Since the structure of $Q$ is known, this usually involves very few multiplications relative to the size of the intensity matrix, which is $K \times K$. Furthermore, only at most $M T$ rows of $P(\Delta)$ need be calculated to estimate the model, corresponding to the number of observations. Algorithms are available which exploit the sparsity of $Q$ and directly compute the action of $P(\Delta)$ on some vector $v$, further reducing the computational cost. Since $v$ can be the $n$-th standard basis vector, one can compute only the necessary rows of $P(\Delta)$.

We now provide some intuition for why discrete-time data will not substantially complicate the problem, which also suggests a natural way to form a simulation-based estimator of $P(\Delta)$. Recall that we are considering stationary models: the hazards do not depend on t. This implies that we can decompose the Markov jump process into two components: a state-independent Poisson process dictating when moves occur, and an embedded Markov chain which dictates where the state moves to.

In our setting, the embedded Markov chain associated with moves by agents is a matrix containing the relevant conditional choice probabilities. We can also rewrite the state transition rates for nature in a similar manner. Namely, let $\bar{q}$ denote the maximum sum of these rates across all states:

$$
\bar{q}=\max _{j} \sum_{k} q_{j k} .
$$

If we consider $\bar{q}$ to be the move arrival rate for nature, then at each move opportunity, the probability of transitioning from state $k$ to $l$ due to a move by nature is

$$
q_{k l}^{\prime}= \begin{cases}q_{k l} / \bar{q} & \text { if } k \neq l \\ 1-\sum_{k \neq l} q_{k l} / \bar{q} & \text { if } k=l\end{cases}
$$

Given the conditional choice probabilities, $\sigma$, and the transition probabilities for nature, $q^{\prime}$, we can construct an embedded Markov chain $\mathcal{Z}$ which characterizes the transition probabilities across all states for each arrival of the alternative Poisson process with rate $\bar{q}+N \lambda$. The transition matrix associated with moving from any state $k$ to any future state $k^{\prime}$ in exactly $r$ steps is simply $\mathcal{Z}^{r}$. Let $a_{n}$ denote a vector of length $K$, which has a one in position $k_{n}$, corresponding to the state at observation $n$, and zeros elsewhere (i.e., the $k_{n}$-th standard basis vector). The maximum likelihood estimates given a dataset of discrete observations at intervals of unit length $(\Delta=1)$ satisfy

$$
(\hat{q}, \hat{\theta})=\arg \max _{(q, \theta)} \sum_{n=1}^{N} \ln \left[\sum_{r=0}^{\infty} \frac{(\bar{q}+N \lambda)^{r} \exp (-(\bar{q}+N \lambda))}{r !} a_{n}^{\top} \mathcal{Z}(q, \theta)^{r} a_{n+1}\right]
$$


The first term in the innermost summation above is the probability of exactly $r$ state changes occurring during a unit interval, under the Poisson distribution. The second term is the probability of the observed state transition, given that there were exactly $r$ moves.

The expression above also suggests a simulation-based estimator. Namely, use the expression inside the sum for the first $R<\infty$ terms. Given an initial guess of $\bar{q}$, draw from the event distribution conditional on having more than $R$ events. One could then use importance sampling to weight the number of events to avoid redrawing the simulated paths when changing the parameters.

\subsubsection{Two-Step Estimation with Discrete Time Data}

We now formalize our assumptions in order to define the two-step estimator with discrete time data and establish its large sample properties. First, following most papers in the literature on two-step estimation (e.g., Bajari et al., 2007, Aguirregabiria and Mira, 2007) we require that the data be generated by a single Markov perfect equilibrium and that all players expect the same equilibrium to be played at all times both in and out of sample. This assumption is analogous to similar assumptions commonly used in two-step estimation of discrete time models (see Aguirregabiria and Mira (2010) for a survey).

Assumption 4. (a) In each market $m=1, \ldots, M$, players expect the same equilibrium, with corresponding intensity matrix $Q$, to be played for all times $t \in[0, \infty)$. (b) The distribution of state transitions in each market $m$ and each time period $t$ is consistent with row $k_{m t}$ of the transition matrix $P(\Delta)=\exp (\Delta Q)$.

As defined in (16), let $h$ denote the vector of all distinct hazards for nature and all state-specific, non-continuation action hazards of players that appear in the matrices $Q_{0}, Q_{1}, \ldots, Q_{N}$. Recall that given any $h \in \mathcal{H}$ a corresponding matrix $Q=\sum_{i=1}^{N} Q_{i}$ can be constructed.

Let $\theta$ denote the finite-dimensional vector of parameters, in some parameter space $\Theta$, which determine the flow payoffs $u$ and instantaneous payoffs $\psi$. Then, let $\Lambda: \Theta \times \mathcal{H} \rightarrow \mathcal{H}$ : $(\theta, h) \mapsto \Lambda(\theta, h)$ denote the mapping which, given vectors $\theta$ and $h$, a new vector $h^{\prime}=\Lambda(\theta, h)$ is determined using the inverse CCP mapping. This proceeds in two steps. First, given $\theta$ and $h$, we construct $Q$ and $\sigma$ and obtain new value functions. In the second step, given the new value functions we determine the new choice probabilities $\sigma^{\prime}$ and then form the new vector $h^{\prime}$. Note that equilibrium conditional choice probabilities must satisfy $h=\Lambda(\theta, h)$.

We note that $\Lambda$ here can be either of two mappings. If the finite dependence property of Proposition 4 holds, $\Lambda$ can be the mapping defined by the representation in (6). More generally, under Proposition $6, \Lambda$ can be the mapping defined by the linear representation of (9) for the infinite-horizon case. Although we have only considered these two cases so far, 
Appendix A provides details on other two-step approaches to estimation of the model such as the forward-simulation-based estimators of Hotz et al. (1994) and Bajari et al. (2007) and the nested pseudo-likelihood estimator of Aguirregabiria and Mira (2007).

The remaining conditions are standard regularity conditions requiring markets to be independent, the parameter space to be compact, the population parameter vector to be identified, and the hazard mapping $\Lambda$ to be sufficiently smooth.

Assumption 5. $\Theta$ is compact and the true parameters $\theta^{0}$ lie in the interior of $\Theta$.

Assumption 6. For any $\theta \in \Theta$ with $\theta \neq \theta^{0}$ and any $h$ such that $h=\Lambda(\theta, h)$, we have $h \neq h^{0}$ for the hazards $h^{0}$ implied by $\theta^{0}$.

Assumption 7. $\Lambda: \Theta \times \mathcal{H} \rightarrow \mathcal{H}:(\theta, h) \mapsto \Lambda(\theta, h)$ is twice continuously differentiable.

Define the pseudo likelihood function

$$
L_{M}(\theta, h)=\frac{1}{M} \sum_{m=1}^{M} \sum_{n=2}^{T} \ln P_{k_{m, n-1}, k_{m n}}(\Delta ; \Lambda(\theta, h)),
$$

where $P_{k, l}(\Delta ; h)$ denotes the $(k, l)$ element of the transition matrix induced by the rates and CCPs in $h$. In the case where the model has a unique equilibrium, we could define the full-solution maximum likelihood estimator as

$$
\begin{array}{r}
\tilde{\theta}=\arg \max _{\theta \in \Theta} L_{M}(\theta, h) \\
\text { s.t. } \quad h=\Lambda(\theta, h) .
\end{array}
$$

A nested fixed point (NFXP) procedure would obtain the equilibrium CCPs (components of $h$ ) for each trial value of $\theta$ given by an optimization routine (Rust, 1987).

Since we have not shown that the equilibrium is unique, we consider a two-step estimation approach under Assumption 4. This estimator has the additional benefit of reducing the computational burden of estimating $\theta^{0}$. Suppose we have a $\sqrt{M}$-consistent first stage M-estimator $\hat{h}$ for $h^{0}$. For example, the nonparametric maximum likelihood estimator of $h$ based on $P(\Delta ; h)$ is an estimator of this kind. We define the feasible two-step pseudo maximum likelihood estimator $\hat{\theta}$ of $\theta^{0}$ as

$$
\hat{\theta}=\arg \max _{\theta \in \Theta} L_{M}(\theta, \hat{h})
$$

Under the maintained assumptions, we show that $\hat{\theta}$ is consistent and asymptotically normal. 
Proposition 7. Suppose that Assumptions 1-7 hold and that $\hat{h}$ is an M-estimator of $h^{0}$ such that $\sqrt{M}\left(\hat{h}-h^{0}\right) \stackrel{\mathrm{d}}{\rightarrow} \mathrm{N}(0, \Sigma)$. Then

$$
\sqrt{M}\left(\hat{\theta}-\theta^{0}\right) \stackrel{\mathrm{d}}{\rightarrow} \mathrm{N}\left(0, \Omega_{\theta \theta^{\top}}^{-1}+\Omega_{\theta \theta^{\top}}^{-1} \Omega_{\theta h^{\top}} \Sigma \Omega_{\theta h^{\top}}^{\top} \Omega_{\theta \theta^{\top}}^{-1}\right)
$$

where $\Omega_{\theta \theta^{\top}}=\mathrm{E}\left[\nabla_{\theta} s_{m} \nabla_{\theta^{\top}} s_{m}\right]$ and $\Omega_{\theta h^{\top}}=\mathrm{E}\left[\nabla_{\theta} s_{m} \nabla_{h^{\top}} s_{m}\right]$ and $s_{m}$ is the pseudo-score

$$
s_{m} \equiv \sum_{t=2}^{T} \ln P_{k_{m, n-1}, k_{m n}}\left(\Delta ; \Lambda\left(\theta^{0}, h^{0}\right)\right) \text {. }
$$

\subsection{Unobserved Heterogeneity}

Our methods can also be extended to accommodate permanent unobserved heterogeneity using finite mixture distributions. In particular, suppose that $T$ observations are sampled at intervals of length $\Delta$ for each of $M$ markets, where each market is one of $Z$ types. Let $\pi_{z}\left(k_{m 1}\right)$ denote the population probability that market $m$ is of type $z$ conditional on the initial observation. ${ }^{13}$ We can then integrate with respect to the distribution of the unobserved state, so that the maximum likelihood problem becomes

$$
(\hat{q}, \hat{\theta}, \hat{\pi})=\arg \max _{(q, \theta, \pi)} \sum_{m=1}^{M} \ln \left[\sum_{z=1}^{Z} \pi_{z}\left(k_{m 1}\right) \prod_{n=2}^{N} \ln P_{k_{m, n-1}, k_{m n}}(\Delta ; q, \theta, z)\right]
$$

where $P(\Delta ; q, \theta, z)$ is the transition matrix for type $z$.

Although (19) is written for the full-solution case, the methods outlined in Arcidiacono and Miller (2011) apply. They show that the EM algorithm can be used to recover the conditional choice probabilities as part of the maximization problem or in a first stage. The same methods can be applied here, only now it is the reduced form hazards conditional on both the observed and unobserved states that are being recovered.

\subsection{Nonparametric Identification}

With continuous-time data, identification and estimation of the intensity matrix for finitestate Markov jump processes is straightforward and well-established (Billingsley, 1961). However, in general when a continuous-time process is only sampled at equispaced points in time, the parameters of the underlying continuous-time model may not be identified. This potential identification problem is also known as the aliasing problem in continuoustime models and it has has been studied by many authors in the context of continuous-time multivariate regression models (e.g., Phillips, 1973, Hansen and Sargent, 1983). ${ }^{14}$ In our

\footnotetext{
${ }^{13}$ By letting $\pi_{z}\left(k_{m 1}\right)$ depend on $k_{m 1}$, we allow for an initial conditions problem.

${ }^{14} \mathrm{~A}$ related issue is the embeddability problem: could the observed discrete-time transition matrix $P(\Delta)$, associated with a time interval of length $\Delta$, have been generated by the proposed data generating process
} 
setting, the question is whether there exists an observationally equivalent continuous time model that could have generated the data and that is also consistent with the structure implied by the dynamic model. We summarize the nonparametric identification results for our model in this section which show that any other continuous time model that could be consistent with the observable data would imply a fundamentally different structure and is therefore not permissible (e.g., a $Q$ matrix containing a non-zero hazard for a transition that is not permissible, or a collection of choice-specific payoffs that violate an exclusion restriction).

In the context of a general finite-state Markov jump processes, the question of identification is whether there a unique matrix $Q$ that leads to the observed transition matrix $P(\Delta)$ when the process is sampled at intervals of length $\Delta$. In closely related work on firstorder systems of stochastic differential equations, Phillips (1973) discusses the role of prior information and gives conditions under which linear restrictions can lead to identification. Blevins (2013) shows that identification can be achieved with even fewer prior restrictions and in the special case of a finite-state Markov jump processes, he gives conditions under which only $\lfloor(K-1) / 2\rfloor$ prior restrictions are needed.

The structural model underlying the $Q$ matrix in our framework provides exactly the sort of prior restrictions needed to mitigate the aliasing problem. The model restricts $Q$ to a lower-dimensional subspace, since it is sparse and must satisfy both within-row and acrossrow restrictions. Therefore, even if there are multiple matrix solutions to the equation $P(\Delta)=\exp (\Delta Q)$, it is unlikely that two of them simultaneously satisfy the restrictions of the structural model. For example, consider the restrictions on the $Q$ matrix implied by the simple renewal model of Section 2.1:

$$
Q=\left[\begin{array}{cccccc}
-q_{1}-q_{2} & q_{1} & q_{2} & 0 & \ldots & 0 \\
\lambda \sigma_{12} & -q_{1}-q_{2}-\lambda \sigma_{12} & q_{1} & q_{2} & \cdots & 0 \\
\lambda \sigma_{13} & 0 & -q_{1}-q_{2}-\lambda \sigma_{13} & q_{1} & \cdots & 0 \\
\vdots & \vdots & \vdots & \ddots & \vdots & \vdots \\
\lambda \sigma_{1, K-1} & 0 & \cdots & 0 & -q_{1}-q_{2}-\lambda \sigma_{1, K-1} & q_{1}+q_{2} \\
\lambda \sigma_{1 K} & 0 & \cdots & 0 & 0 & -\lambda \sigma_{1 K}
\end{array}\right]
$$

The number of parameters to be estimated in this matrix is substantially less than if the

(some continuous-time Markov structure with intensity matrix $Q$ or some discrete-time chain over fixed time periods of length $\delta$ ). This is a model specification issue, also arising in both discrete time and continuous time: was the data actually generated by a continuous time Markov jump process? We assume throughout that the model is well-specified and therefore, such an intensity matrix $Q$ exists. Singer and Spilerman (1976) provide several necessary conditions for embeddability involving testable conditions on the determinant and eigenvalues of $P(\Delta)$. This problem was first proposed by Elfving (1937). Kingman (1962) derived the set of embeddable processes with $K=2$ and Johansen (1974) gave an explicit description of the set for $K=3$. 
intensities were allowed to vary across all the non-diagonal elements. In fact, there are $K^{2}-$ $4 K+8$ zero restrictions while under the conditions of Blevins (2013) as few as $\lfloor(K-1) / 2\rfloor$ are sufficient for identification of $Q$ given $P(\Delta)$. Furthermore, the CCPs depend on the rates and payoff parameters, which introduces shape restrictions on $\sigma_{1 k}$ across states $k$.

In general, as in this example, a large number of zero restrictions are typically implied naturally by the model specification. Blevins (2014) shows that for models of the kind we consider, all non-trivial binary-choice $(J=1)$ games are identified using only these zero restrictions. More generally, when there is a player-specific state variable that can take $\kappa$ values, then any model is identified as long as the number of choices is not too large: $J \leq\left(\kappa^{N}-1\right) /(2 N)$. Other (non-zero) restrictions can also be added. For example, if continuous-time data is available on a subset of the state variables, then certain elements of $Q$ are identified and can serve as restrictions.

Once identification of $Q$ has been established given $P(\Delta)$, the question remains as to whether the choice probabilities, value functions, choice-specific payoffs, and flow payoffs are also identified. Although we do not consider these details here, Blevins (2014) also gives conditions for which $\sigma_{i k}, V_{i k}$, and $u_{k}$ are nonparametrically identified. Possible identifying restrictions on $V_{i k}$ and $\psi_{i k}$ include payoff exclusion restrictions and equality restrictions on the choice-specific payoffs across states.

Importantly, the question of identification applies equally to discrete-time models, where there is an analogous time-aggregation problem (Bajari, Hong, and Nekipelov, 2013, Hong, $\mathrm{Li}$, and Wang, 2012). Suppose there is a fixed move interval of length $\delta$ in the model which may be different from the fixed interval $\Delta$ at which observations are sampled. In practice, researchers typically assume (implicitly) that $\delta=\Delta$, where $\Delta=1$ is normalized to be some specific unit of time (e.g., one quarter).This assumption is convenient, but masks the identification problem, which requires that there exist a unique matrix root $P_{0}$

of the discrete-time aggregation equation $P_{0}^{\Delta / \delta}=P(\Delta)$. In general, however, there may be multiple such matrices (Singer and Spilerman, 1976, p. 49). As in our setting, however, valid solutions $P_{0}$ must satisfy the restrictions implied by the model. These issues become trivial under the usual assumption that $\delta=\Delta$.

\subsection{Comparison to Discrete Time Methods}

Characterizing dynamic problems in continuous time has both advantages and disadvantages when compared to discrete time formulations.

From a computational perspective, the key benefit to working in continuous time concerns the treatment of counterfactuals. Here there are two advantages over discrete time. First, even if the data is time aggregated, the counterfactual analysis can leverage the full computational benefit of the underlying continuous time process. In particular, instanta- 
neous representations of the value functions can be used to solve for counterfactual hazards, greatly reducing computational times because only one event occurs in any given instant. In the typical discrete time set up, all players move simultaneously, sharply increasing the computational burden. Second, one source of multiple equilibria - simultaneous moves - is eliminated in the continuous time context. Multiple equilibria make interpreting counterfactuals difficult due to ambiguity regarding which equilibrium would be played in the counterfactual environment.

On the other hand, estimation is more complicated in continuous time when data are time aggregated, as it requires integrating out over the possible paths between the observed state changes. It is important to keep in mind, however, that estimation is not the main bottleneck for research, as consistent estimates can be obtained with two-step estimation in either discrete or continuous time with low computational burden.

Continuous and discrete time representations of dynamic games also have different economic implications. While continuous time methods may capture the underlying decisionmaking and strategy interaction well in many setting, they may be a poor fit for some research problems. First, it may be the case that a simultaneous move environment and the resulting coordination failures are a salient feature of the economic setting. For example, the adoption of new technology standards may be delayed by a failure by incumbent players to quickly agree on a standard. Second, the methods developed here apply to stationary environments. While dynamic games are typically estimated assuming stationarity, there are exceptions. See Beauchamp (2014) and Igami (2014) for examples of empirical games in non-stationary environments. Further work is necessary to understand whether the tools developed here can be extended to non-stationary, continuous time environments.

Finally, conceptual issues may dictate the choice between discrete and continuous time. It is well-known that in a discrete time setting with asymmetric information, agents can sometimes make "mistakes" that arise from the simultaneity of choice (for example, simultaneously opening in the same location as a rival with a low ex-ante probability of entering because the rival happened to receive a "high" idiosyncratic shock). This is obviously most damaging in a static model (where firms cannot correct their mistakes) but may be unattractive in a dynamic setting as well if, for example, the choice requires substantial investments to un-wind. That said, if such "mistakes" are a salient feature of the economic environment because, for example, firms only observe their rival's actions with a lag, a discrete time model might be able to capture this behavior more simply than a continuous time model.

More broadly, the regularity of decision timing implied by the discrete time model may be preferred in some settings. For example, there may be institutional features that restrict the timing of when decisions can be made. Board meetings may occur at pre-specified (e.g. quarterly) times during the year or long-term contracts (or implicit agreements) might 
prevent an agent from changing its actions for a pre-specified period of time. In such cases, a discrete time model may be more appropriate. On the other hand, a discrete time model may force many outcomes to occur at the same instant that are more naturally viewed as occurring sequentially (e.g. the opening or closing of stores, changes in installed capacity, or the release of new products). Generally, the appropriate choice of modeling assumption will depend on the institutional setting being considered.

\section{Wal-Mart's Entry into the Supermarket Industry}

Our empirical application considers the impact of Wal-Mart's entry into the supermarket industry. In 1994, the first year for which we have data, Wal-Mart owned 97 supercenter outlets. However, by 2006, the last year of our data, they operated 2225 such outlets and ranked first among all grocery firms in terms of overall sales. Much of this expansion came at the expense of incumbent grocers. The question is exactly which types of firms were most impacted and how the competitive landscape evolved in response.

Wal-Mart first gained national prominence through its gradual rise to an ultimately dominant position in the discount store (general merchandise) industry. Due to its large role in the U.S. economy (Wal-Mart accounted for 8.8 percent of (non-automobile) retail sales in 2004), Wal-Mart has attracted significant attention from both the popular press and academic researchers. Much of the debate centers on Wal-Mart's overall impact on consumer surplus, labor market outcomes, and local competitors. Our focus here will be on its impact on competition and market structure.

There is no doubt that Wal-Mart has had a significant impact on retail competition. Due to its scale and operational efficiency, Wal-Mart is often able to undercut the prices of its rivals, both in general merchandise and groceries. For example, in the context of groceries, Basker and Noel (2009) find that Wal-Mart is able to set prices that are on average 10 percent lower than their competitors and that this differential appears to be increasing over time. They also find evidence of a competitive response: in the short run, competing grocery stores reduce prices by $1-2 \%$ when a Wal-Mart enters. They note that the response is mostly due to smaller scale competitors - the reaction by the top 3 national chains is only half as large. Matsa (2011) looks at Wal-Mart's impact on supermarket stock-outs (a measure of quality) and finds that entry by Wal-Mart decreases stock-outs at competing supermarkets, but that this impact is instead centered on large-scale competitors. The

smaller rivals either cut prices or exit. Since much of Wal-Mart's advantage appears to derive from its enormous scale and intensive investment in information technology (Basker, 2007), there is particular concern that Wal-Mart stifles small scale entrepreneurial activity. This was particularly salient in the context of the discount store industry (Wal-Mart's 
original line of businesss) which was, prior to entry by Wal-Mart, served by a collection of single-store outlets that typically focused on a more narrow line of goods. By offering greater breadth and depth of assortment, Wal-Mart consistently leveraged its greater scale to undercut prices and consolidate purchases (by offering one stop shopping). The impact on small scale firms was unambiguously negative - Wal-Mart displaced the "mom and pop" stores. Jia (2008) concludes that Wal-Mart's expansion alone drove 50-70 percent of the net exit of small discount retailers from the late 1980s to mid 1990s. Focusing on the big box industry as a whole, Haltiwanger, Jarmin, and Krizan (2010) find a large negative impact of big box chains (including Wal-Mart) on single store retailers and small chains, continuing a long term trend toward larger chains throughout much of retail.

However, Wal-Mart's impact on the structure of the grocery industry is less clear. While Matsa (2011) finds a significant negative impact on the survival probabilities of small-scale rivals consistent with earlier experience in the discount industry (and a shift up market by the larger chains), Ellickson and Grieco (2013) find a large, geographically localized negative impact of Wal-Mart centered on the large grocery chains, with no measurable impact on the small firms at all. In the analysis presented below, we find that Wal-Mart's negative impact falls almost entirely on the large chains and is actually associated with an expansion by the single store segment. This sharp contrast with the earlier case of the discount segment is striking, and illustrates the benefit of a dynamic structural model as the overall shift is an equilibrium result that evolves slowly over time.

\subsection{Data}

Our data for the supermarket industry are drawn from yearly snapshots of the Trade Dimensions Retail Database, capturing the set of players that are active in September of each year, starting in 1994 and ending in 2006. Trade Dimensions continuously collects information on every supermarket (and many other retailers) operating in the United States for use in their Marketing Guidebook and Market Scope publications and as a standalone, syndicated dataset. The definition of a supermarket used by Trade Dimensions is the government and industry standard: a store selling a full line of food products that grosses at least $\$ 2$ million in revenue per year. Store level data on location and a variety of in-store features is linked to the firm level through a firm identity code, which can also be used to identify the location of the nearest distribution facility. In addition to the Trade Dimensions data, which consists of yearly snapshots of the entire industry, we also have information on the exact opening dates of the Wal-Mart supercenters that were gathered from a variety of online sources.

Although not strictly necessary to our methods being able to handle time aggregation, for some of our specifications we use data on the exact date of Wal-mart entry. This data 
comes from two sources. First is data downloaded from trendresults.com which gives the opening date for all Wal-Mart stores. Some stores, however, were converted from retail stores to supercenters and this data misses these conversion dates. This is a particular issue earlier in the sample. We supplement this data with data from Emek Basker who was provided with entry dates up through 2005, though some of the dates were clustered around particular days suggesting time aggregation in some cases. We only used exact Wal-mart entry dates if we were confident in the timing. ${ }^{15}$

For market definition, we focus on Metropolitan Statistical Areas (MSAs) with population under 500,000, yielding a total of 309 markets. For our purposes, a firm is deemed to be a chain firm in a market if it has at least 20 stores open nationally and its maximum market share (in terms of number of stores) exceeds $20 \%$ in at least one year. We allow for up to seven chain players in each MSA who may or may not be active in the market at any given time. If a chain has no stores in a particular period and chooses not to build a store, that chain is replaced by a new potential chain entrant. In our model, we allow for ten potential fringe firm entrants in each MSA, so the number of fringe firms is the number of incumbent fringe firms plus ten. ${ }^{16}$

Demand for supermarkets is a function of population. The data on market population are interpolated from the decennial censuses of the United States and population is discretized into six categories. ${ }^{17}$ Each MSA is assigned to one of three population growth categories based on the change in the population of the MSA over the full sample period. In particular, the growth category of a city is fast if the annual growth rate is greater than $2 \%$ ( 74 cities), moderate if the annual growth rate is between $1 \%$ and $2 \%$ (106 cities), and slow if the annual growth rate is less than $1 \%$ (129 cities). The parameters governing population transitions are indexed by these growth categories.

Table 1 gives descriptive statistics for the sample. On average, there are about two and a half chain firms per market, with 3.7 stores per chain firm on average. Markets contain an average of 13 fringe stores. The number of Wal-Marts is much smaller, averaging one store per market in the sample. On average, there are $0.277,0.177$, and 0.825 stores built per market within a year by chain firms, Wal-Mart, and fringe firms, respectively. The corresponding figures for store closings are $0.224,0.002$, and 0.908, revealing that Wal-Mart virtually never exits in our sample.

Table 2 looks at entry and exit decisions for chain firms and fringe firms one year before,

\footnotetext{
${ }^{15}$ In total we use 256 for dates for 475 Wal-mart entries. The match rate is much higher in later years, with exact dates on 213 of 260 entries between 2001 and 2006.

${ }^{16}$ Since we are treating the firm/market as the relevant unit of analysis, we do not model situations in which a supermarket chain moves one of their stores to another location within the market. In such cases, we consider only the net effect, meaning that we assume that if we see both an entry and exit by the same chain, this pair of moves is equivalent to not moving at all.

${ }^{17}$ The discretization was such that differences in log population between adjacent categories was equal.
} 
the year of, and the year after initial entry of Wal-Mart. Here, we see that chain and fringe firms both respond negatively to Wal-Mart. The number of new chain stores falls from 0.311 in the period before Wal-Mart enters to 0.189 in the period after - a $40 \%$ drop. Similarly, the number of stores that close increases by over $6.5 \%$ from a base level of 0.122 . The qualitative patterns for fringe firms are the same, though the effects are muted, suggesting that Wal-Mart's presence is more detrimental to chain firms than fringe firms.

This table highlights an advantage of using a model where the frequency of moves can differ from the data sampling frequency. Note that the numbers of entering and exiting chain stores in the year of Wal-Mart's initial entry are bracketed by the corresponding values the year before and the year after Wal-Mart's entry. In markets where chain and fringe stores saw little change in their building patterns, this suggests that Wal-Mart entered later in the period. But when Wal-Mart enters early in the period, exit by chain and fringe stores is more likely to occur within the period. Our model can explain both the increase in exit rates during the entry period as well as the additional increase in exit rates in the period after.

\subsection{Model}

To quantify Wal-Mart's impact on large versus small rivals, and allow for heterogeneous competitive effects across firm types, there are three types of firms in our model: chain firms (who can operate many stores), Wal-Mart (who can also operate many stores), and fringe firms (who can operate at most one store each). We assume that the chain firms (including Wal-Mart) make strategic decisions within each local market (MSA), but independent decisions across markets (i.e. they do not choose their entire spatial layout jointly, but rather make optimal decisions on a market by market basis). With each move arrival, chain stores can open one new store $(j=1)$, do nothing $(j=0)$, or, conditional on having at least one open store, close a store $(j=-1)$. A move arrival for an incumbent fringe firm provides an opportunity for the firm to exit. Similarly, move arrivals provide opportunities for potential entrants to enter. In the context of retail competition, a random move arrival process might reflect the stochastic timing of local development projects (e.g., housing tracts and business parks), delays in the zoning and permitting processes, and the random arrival of retailers in other lines of business that have higher valuations for the properties currently occupied by incumbent grocers. All firms have the same move arrival rate, normalized at $\lambda=1$, and $q_{1}$ and $q_{-1}$ are the rates of moving up and down in population, respectively.

Our model is a continuous-time, discrete action version of the dynamic oligopoly model of Ericson and Pakes (1995) and Pakes and McGuire (1994), in which heterogeneous firms make entry, exit, and investment decisions. Firms in our model are differentiated by type (Wal-Mart, chain, or fringe) and by the number of stores they operate. Firms invest by 
building new stores and disinvest by closing stores.

Since the state variables are discrete, we enumerate all possible states by an integer scalar index $k=1, \ldots, K$. The state of the market at each instant can be summarized by a vector $x_{k}$ containing the number of stores operated by each chain firm, $s_{1 k}^{\mathrm{c}}, s_{2 k}^{\mathrm{c}}, \ldots$, each fringe firm, $s_{1 k}^{\mathrm{f}}, s_{2 k}^{\mathrm{f}}, \ldots$, and Wal-Mart, $s_{k}^{\mathrm{w}}$, along with the current population, $d_{k}$ :

$$
x_{k}=\left(s_{1 k}^{\mathrm{c}}, s_{2 k}^{\mathrm{c}}, \ldots, s_{1 k}^{\mathrm{f}}, s_{2 k}^{\mathrm{f}}, \ldots, s_{k}^{\mathrm{w}}, d_{k}\right)
$$

Therefore, each value of $k$ represents an encoded state vector and the function $l(i, j, k)$ gives the state conditional on firm $i$ taking action $j$ in state $k$. Additionally, each market is characterized by a time-invariant unobserved type $z$, which is observed by the firms in the market but not by the econometrician. ${ }^{18}$ Hence, the full state vector at any instant can be written as $\left(x_{k}, z\right)$.

\subsubsection{Value Functions}

We now provide the general formulation of the value functions and then describe the relevant state variables. For a particular market, the value function for firm $i$ in state $k$ is given by:

$V_{i k}=\frac{u_{i k}+\sum_{j \in\{-1,1\}} q_{j} V_{i, l(0, j, k)}+\sum_{m \neq i} \lambda \sum_{j \in\{-1,1\}} \sigma_{m j k} V_{i, l(m, j, k)}+\lambda \operatorname{Emax}_{j}\left\{V_{i, l(i, j, k)}+\psi_{i j k}+\varepsilon_{i j k}\right\}}{\rho+\sum_{j \in\{-1,1\}} q_{j}+\sum_{m \neq i} \lambda \sum_{j \in\{-1,1\}} \sigma_{m j k}+\lambda}$.

In this expression, nature is indexed by $i=0$, the choices are $j=1$ and $j=-1$, and the costs $\psi_{i j k}$ reflect the costs of initial entry, building new stores, or closing stores depending on the values of the player identity $i$, choice $j$, and state $k$. We will fully specify these costs for each type of firm below.

Following standard convention in the empirical entry literature, we assume that if a chain or fringe firm closes all of its stores, then the firm cannot enter again later (in effect, the continuation value for exit is identically zero). Hence, if a chain firm exits, it would be replaced by a new potential chain entrant. For chain and fringe firms, this allows us to replace the value functions on the right-hand side of (20) using Propositions 2 and 3. As a result (and exploiting Proposition 4), the value function on the left-hand side of (20) can be expressed as a function of the flow payoffs, the move arrival parameters, and the probabilities of making particular decisions. Because Wal-Mart essentially never exits or closes stores, we cannot recover their full set of structural parameters. Nonetheless, we are able to fully account for their strategic impact on rivals' actions by using our first stage estimates to capture their rivals' beliefs about Wal-Mart's equilibrium policy functions.

\footnotetext{
${ }^{18}$ We use $Z=5$ points of support, $z \in\{-1.3998,-0.5319,0.0,0.5319,1.3998\}$, based on a discrete approximation to a standard normal random variable.
} 
This flexibility is an additional advantage of using a two-step estimation approach.

\subsubsection{Flow Profits and Choice-Specific Payoffs for Chain Firms}

We specify the flow payoff $u_{i k}$ for chain firms in terms of per-store latent revenue and total cost. These are linear functions of market population, $d_{k}$, the number of own stores, $s_{i k}^{\mathrm{c}}$, the number of competing chain stores, $\tilde{s}_{i k}^{\mathrm{c}}$, the number of Wal-Mart stores, $s_{k}^{\mathrm{w}}$ (WalMart), and the number of fringe stores, $s_{k}^{f}$. Revenues also depend on an unobserved (to the econometrician) characteristic of the market, $z$, which reflects the tastes of consumers in a given market for particular types of products. Flow profits for a chain firm $i$ in state $k$ are

$$
u_{i k}^{\mathrm{c}}=s_{i k}^{\mathrm{c}}\left(\beta_{0}^{\mathrm{c}}+\beta_{1}^{\mathrm{c}} \tilde{s}_{i k}^{\mathrm{c}}+\beta_{2}^{\mathrm{c}} s_{k}^{\mathrm{w}}+\beta_{3}^{\mathrm{c}} s_{k}^{\mathrm{f}}+\beta_{4}^{\mathrm{c}} s_{i k}^{\mathrm{c}}+\beta_{5}^{\mathrm{c}} d_{k}+\beta_{6}^{\mathrm{c}} z+\beta_{7}^{\mathrm{c}} z s_{i k}^{\mathrm{c}}\right)+e_{i k}^{\mathrm{c}},
$$

where $e_{\mathrm{c} i k}$ is (the negative of) the flow cost of operating a set of stores:

$$
e_{i k}^{\mathrm{c}}=\mu_{1}^{\mathrm{c}} s_{i k}^{\mathrm{c}}+\mu_{2}^{\mathrm{c}}\left(s_{i k}^{\mathrm{c}}\right)^{2}+\mu_{3}^{\mathrm{c}}\left(s_{i k}^{\mathrm{c}}\right)^{3} .
$$

A cubic cost function allows there to be regions of increasing and then decreasing returns to scale, so that for each state the optimal value of $s_{i k}^{c}$ is finite. Expanding and collecting terms yields

$$
\begin{aligned}
u_{i k}^{\mathrm{c}} & =s_{i k}^{\mathrm{c}}\left(\left(\beta_{0}^{\mathrm{c}}+\mu_{1}^{\mathrm{c}}\right)+\beta_{1}^{\mathrm{c}} \tilde{s}_{i k}^{\mathrm{c}}+\beta_{2}^{\mathrm{c}} s_{k}^{\mathrm{w}}+\beta_{3}^{\mathrm{c}} s_{k}^{\mathrm{f}}+\left(\beta_{4}^{\mathrm{c}}+\mu_{2}^{\mathrm{c}}\right) s_{i k}^{\mathrm{c}}+\mu_{3}^{\mathrm{c}}\left(s_{i k}^{\mathrm{c}}\right)^{2}+\beta_{5}^{\mathrm{c}} d_{k}+\beta_{6}^{\mathrm{c}} z+\beta_{7}^{\mathrm{c}} z s_{i k}^{\mathrm{c}}\right) \\
& =s_{i k}^{\mathrm{c}}\left(\theta_{0}^{\mathrm{c}}+\theta_{1}^{\mathrm{c}} \tilde{s}_{i k}^{\mathrm{c}}+\theta_{2}^{\mathrm{c}} s_{k}^{\mathrm{w}}+\theta_{3}^{\mathrm{c}} s_{k}^{\mathrm{f}}+\theta_{4}^{\mathrm{c}} s_{i k}^{\mathrm{c}}+\theta_{5}^{\mathrm{c}}\left(s_{i k}^{\mathrm{c}}\right)^{2}+\theta_{6}^{\mathrm{c}} d_{k}+\theta_{7}^{\mathrm{c}} z+\theta_{8}^{\mathrm{c}} z s_{i k}^{\mathrm{c}}\right) .
\end{aligned}
$$

The choice-specific instantaneous payoffs $\psi_{i j k}$ depend on the unobserved state $z$ and differ according to whether firm $i$ is an incumbent $\left(s_{i k}^{\mathrm{c}}>0\right)$ or new entrant $\left(s_{i k}^{\mathrm{c}}=0\right)$ and whether the choice is building a new store $(j=1)$ or closing an existing store $(j=-1)$ :

$$
\psi_{i j k}= \begin{cases}\eta_{0}^{\mathrm{c}}+\eta_{1}^{\mathrm{c}} z+\kappa_{0}^{\mathrm{c}}+\kappa_{1}^{\mathrm{c}} z & \text { if } s_{i k}^{\mathrm{c}}=0 \text { and } j=1 \\ \kappa_{0}^{\mathrm{c}}+\kappa_{1}^{\mathrm{c}} z & \text { if } s_{i k}^{\mathrm{c}}>0 \text { and } j=1 \\ \phi_{0}^{\mathrm{c}}+\phi_{1}^{\mathrm{c}} z & \text { if } s_{i k}^{\mathrm{c}}>0 \text { and } j=-1 \\ 0 & \text { otherwise. }\end{cases}
$$

Therefore, the structural parameters of interest for chain firms are the coefficients of the per-store payoff function and the parameters of the instantaneous payoffs:

$$
\theta^{\mathrm{c}}=\left(\theta_{0}^{\mathrm{c}}, \ldots, \theta_{8}^{\mathrm{c}}, \eta_{0}^{\mathrm{c}}, \eta_{1}^{\mathrm{c}}, \kappa_{0}^{\mathrm{c}}, \kappa_{1}^{\mathrm{c}}, \phi_{0}^{\mathrm{c}}, \phi_{1}^{\mathrm{c}}\right)
$$

We assume that there are seven chain firms in all markets with the number of potential 
entrants in a market equal to seven minus the observed chain firms in the market. The twostep estimation we employ means we do not need to place any restrictions on the number of chain stores a firm operates, though we will need to do so when we solve for counterfactual choice probabilities.

\subsubsection{Flow Profits and Choice-Specific Payoffs for Fringe Firms}

Flow profits for fringe stores have a similar linear form to that of chain firms, though with different coefficients and a different flow cost function, $e_{i k}^{\mathrm{f}}$. Namely, an operating fringe store has profits given by: ${ }^{19}$

$$
u_{i k}^{\mathrm{f}}=\beta_{0}^{\mathrm{f}}+\beta_{1}^{\mathrm{f}} s_{k}^{\mathrm{c}}+\beta_{2}^{\mathrm{f}} s_{k}^{\mathrm{w}}+\beta_{3}^{\mathrm{f}} s_{k}^{\mathrm{f}}+\beta_{4}^{\mathrm{f}} d_{k}+\beta_{5}^{\mathrm{f}} z+\beta_{6}^{\mathrm{f}} z s_{k}^{\mathrm{f}}+e_{i k}^{\mathrm{f}},
$$

Fringe competitors often rely on the same suppliers to deliver goods to their stores. Hence, there may be some density economies present at first. However, at some point competitive influences will drive up costs, suggesting a quadratic cost function in the total number of fringe stores:

$$
e_{i k}^{\mathrm{f}}=\mu_{0}^{\mathrm{f}}+\mu_{1}^{\mathrm{f}} s_{k}^{\mathrm{f}}+\mu_{2}^{\mathrm{f}}\left(s_{k}^{\mathrm{f}}\right)^{2} .
$$

Collecting terms yields the flow profit function

$$
\begin{aligned}
u_{i k}^{\mathrm{f}} & =\left(\beta_{0}^{\mathrm{f}}+\mu_{0}^{\mathrm{f}}\right)+\beta_{1}^{\mathrm{f}} s_{k}^{\mathrm{c}}+\beta_{2}^{\mathrm{f}} s_{k}^{\mathrm{w}}+\left(\beta_{3}^{\mathrm{f}}+\mu_{1}^{\mathrm{f}}\right) s_{k}^{\mathrm{f}}+\mu_{2}^{\mathrm{f}}\left(s_{k}^{\mathrm{f}}\right)^{2}+\beta_{4}^{\mathrm{f}} d_{k}+\beta_{5}^{\mathrm{f}} z+\beta_{6}^{\mathrm{f}} z s_{k}^{\mathrm{f}} \\
& =\theta_{0}^{\mathrm{f}}+\theta_{1}^{\mathrm{f}} s_{k}^{\mathrm{c}}+\theta_{2}^{\mathrm{f}} s_{k}^{\mathrm{w}}+\theta_{3}^{\mathrm{f}} s_{k}^{\mathrm{f}}+\theta_{4}^{\mathrm{f}}\left(s_{k}^{\mathrm{f}}\right)^{2}+\theta_{5}^{\mathrm{f}} d_{k}+\theta_{6}^{\mathrm{f}} z+\theta_{7}^{\mathrm{f}} z s_{k}^{\mathrm{f}} .
\end{aligned}
$$

Recall that fringe firms can operate at most one store. Therefore, potential fringe entrants only choose whether to enter (and build a single store) or not and incumbent fringe firms only decide whether to close their store or not. We assume there are always ten potential fringe entrants. Therefore, the choice-specific instantaneous payoffs for fringe firms, $\psi_{i j k}$, represent entry costs for new entrants (for which $s_{i k}^{\mathrm{f}}=0$ ) and exit values for incumbents (for which $s_{i k}^{\mathrm{f}}=1$ ). Since fringe firms can operate at most one store, we cannot distinguish the entry cost from the building cost. Because we estimate a fixed flow cost parameter for fringe firms, $\theta_{0}^{\mathrm{f}}$, we normalize the exit value to zero. As with chain firms, we allow the entry cost to depend on the unobserved state $z$ :

$$
\psi_{i j k}= \begin{cases}\eta_{0}^{\mathrm{f}}+\eta_{1}^{\mathrm{f}} z & \text { if } s_{i k}^{\mathrm{f}}=0 \text { and } j=1 \\ 0 & \text { otherwise. }\end{cases}
$$

\footnotetext{
${ }^{19}$ Note that there is no term for own number of stores here as fringe stores can only operate at most one store.
} 
Therefore, the structural parameters of interest for fringe firms are the coefficients of the payoff function and the parameters of the instantaneous payoffs:

$$
\theta^{\mathrm{f}}=\left(\theta_{0}^{\mathrm{f}}, \ldots, \theta_{7}^{\mathrm{f}}, \eta_{0}^{\mathrm{c}}, \eta_{1}^{\mathrm{c}}\right)
$$

\subsection{Estimation}

We estimate the model in two steps, first estimating the reduced form hazards that capture the rate of change in the number of stores of each format and the change in population over time, and then estimating the structural parameters of the profit functions, taking the reduced form hazards as given.

\subsubsection{Step 1: Estimating Reduced-Form Hazards}

We estimate the probabilities of opening a store, closing a store (if the firm has at least one store), and doing nothing using a linear-in-parameters multinomial logit sieve, with the parameters varying by firm type (chain, Wal-Mart, and fringe). ${ }^{20}$ In particular, let $\tilde{p}_{i j}(k, z, \alpha)$ denote the reduced form probability of firm $i$ making choice $j$ in state $(k, z)$, which has the form

$$
\tilde{p}_{i j}(k, z, \alpha)=\frac{\exp \left(h_{j}(k, z, \alpha)\right)}{\sum_{j^{\prime} \in \mathcal{A}_{i k}} \exp \left(h_{j^{\prime}}(k, z, \alpha)\right)},
$$

where $h_{j}(k, z, \alpha)$ is a flexible function of the state variables with finite dimensional parameter vector $\alpha$. The likelihood of a particular event, choice $j$ by firm $i$ in state $k$, in a market with unobserved state $z$, with an interval of length $\tau$ since the previous event, is

$$
\lambda \tilde{p}_{i j}(k, z, \alpha) \exp \left[-\left(\sum_{j^{\prime} \in\{-1,1\}} q_{j^{\prime}}+\sum_{m} \lambda \sum_{j^{\prime} \in \mathcal{A}_{m k}} \tilde{p}_{m j^{\prime}}(k, z, \alpha)\right) \tau\right] .
$$

Since we have annual data, we simulate potential sequences of events that can happen over the course of each year. As discussed earlier, the structure of our data is such that we observe all events that took place in each year, but do not observe the exact times at which these events occur. Suppose that we observe $W$ events in period $n$. For periods with $W>0$, we draw $R$ simulated paths, randomly assigning each observed event to a simulated time. Once we have the likelihood of each simulated sequence of events, we average over these simulated sequences, integrating over move times.

\footnotetext{
${ }^{20}$ The variables included in the multinomial logit models are the number of fringe stores and its square, the number of chain stores and its square, the number of Wal-Marts and its square, and the total number of firms squared, and interactions of each of these variables with population. In addition, we control for city growth type, the unobserved state, and the unobserved state interacted with an indicator for building a new store.
} 
Focusing on a particular observation period $n$, let $k_{n-1}$ and $k_{n}$ denote the states at the beginning and end of the period. Let $k_{w}^{(r)}$ denote the state immediately preceding event $w$ in simulation $r$, with $w=1, \ldots, W+1$. The observed state at the beginning of the period is then $k_{1}^{(r)}$, so $k_{1}^{(r)}=k_{n-1}$ for each $r$. Similarly, the terminal state for each path is the observed state at the end of the period, so $k_{W+1}^{(r)}=k_{n}$ for each $r$. Let $I_{w}^{(r)}(i, j)$ be the indicator for whether event $w$ of the $r$-th simulation was action $j$ taken by firm $i$. Conditional on knowing the unobserved state $z$, the simulated likelihood of observation $n$ in market $m$ is

$$
\begin{aligned}
\tilde{L}_{m n}(q, \alpha ; z)=\frac{1}{R} \sum_{r=1}^{R}\left\{\prod_{w=1}^{W}\left[\sum_{j \in\{-1,1\}} I_{w}^{(r)}(0, j) q_{j}+\sum_{i} \lambda \sum_{j \neq 0} I_{w}^{(r)}(i, j) \tilde{p}_{i j}\left(k_{w}^{(r)}, z, \alpha\right)\right]\right. \\
\left.\times \exp \left[-\left(\sum_{j \in\{-1,1\}} q_{j}+\sum_{i} \lambda \sum_{j \neq 0} \tilde{p}_{i j}\left(k_{w}^{(r)}, z, \alpha\right)\right) \tau_{w}^{(r)}\right]\right\} \\
\times \exp \left[-\left(\sum_{j \in\{-1,1\}} q_{j}+\sum_{i} \lambda \sum_{j \neq 0} \tilde{p}_{i j}\left(k_{W+1}^{(r)}, z, \alpha\right)\right)\left(1-t_{W}^{(r)}\right)\right],
\end{aligned}
$$

where $W$ is the number of events that occurred in the $n$-th interval and $t_{W}^{(r)}$ is the time of the last simulated move.

Since $z$ is unobserved, we estimate the reduced form hazards using mixture distributions. Higher values of the unobserved state may make it easier or harder to operate as a chain, fringe, or Wal-Mart store respectively. We discretize the standard normal distribution into five points and then estimate the population probabilities of being at each of these points. Note that there is an initial conditions problem here, so we allow the prior probability of being in a particular unobserved state to depend on the first period state variables, similar to Keane and Wolpin (1997) and Arcidiacono, Sieg, and Sloan (2007). In particular, we specify the prior probabilities as following an ordered logit that depends on the number of chain stores, the number of Wal-Marts, and the number of fringe stores, all interacted with population, and the city growth type.

Let $\pi_{z}\left(k_{1}\right)$ be the probability of the unobserved state being $z$ given that the observed state was $k_{1}$ for the first observation. With $M$ markets and $T$ periods each, integrating with respect to the distribution of the unobserved market states yields

$$
(\tilde{q}, \tilde{\alpha}, \tilde{\pi})=\arg \max _{(q, \alpha, \pi)} \sum_{m=1}^{M} \ln \left(\sum_{z} \pi_{z}\left(k_{m 1}\right) \prod_{n=1}^{N} \tilde{L}_{m n}(q, \alpha ; z)\right) .
$$

The first stage estimates then give both the reduced form hazards, which are subsequently used in the second stage to form the value functions, as well as the conditional probability 
of each market being in each of the unobserved states, as in Arcidiacono and Miller (2011).

\subsubsection{Step 2: Estimating the Structural Parameters}

In Step 2, we take the probabilities of being in each unobserved state and the reduced-form hazards from Step 1 as given. We then separately estimate the structural parameters for chain firms and fringe stores. As noted above, Wal-Mart's limited set of realized actions precludes us from estimating their structural parameters. However, their strategic impact is captured by the first stage estimates, which reflect their rivals' beliefs over Wal-Mart's expected actions. Our counterfactual experiments will then include only scenarios in which Wal-Mart's structural parameters do not play a role (e.g. equilibria in which Wal-Mart no longer exists). Let $P_{m}(z)$ denote the probability of MSA $m$ being in unobserved state $z$ given the data. Using Bayes' rule, we have

$$
P_{m}(z)=\frac{\pi_{z}\left(k_{m 1}\right) \prod_{n=1}^{N} \tilde{L}_{m n}(\tilde{q}, \tilde{\lambda}, \tilde{\alpha} ; z)}{\sum_{z^{\prime}} \pi_{z^{\prime}}\left(k_{m 1}\right) \prod_{n=1}^{N} \tilde{L}_{m n}\left(\tilde{q}, \tilde{\lambda}, \tilde{\alpha} ; z^{\prime}\right)} .
$$

These probabilities are then used as weights in the likelihood function for Step 2.

Next, using Proposition 4, we can express the value function in (20) as a function of the structural parameters, $\theta$, and the reduced form hazards from the first stage, $\tilde{p}_{i j}(k, z, \tilde{\alpha})$. Thus, let $\tilde{\sigma}_{i j}(k, z, \theta)$ denote the implied probability that firm $i$ takes action $j$ in state $(k, z)$, for a given value of the second stage parameters.

Define $\check{L}_{m n}(\theta ; \tilde{q}, \tilde{\alpha} ; z)$ as

$$
\begin{aligned}
\check{L}_{m n}(\theta ; \tilde{q}, \tilde{\alpha}, z)= & \frac{1}{R} \sum_{r=1}^{R}\left\{\prod_{w=1}^{W}\left[\sum_{j \in\{-1,1\}} I_{w}^{(r)}(0, j) q_{j}+\sum_{i} \lambda \sum_{j \neq 0} I_{w}^{(r)}(i, j) \tilde{\sigma}_{i j}\left(k_{w}^{(r)}, z, \theta\right)\right]\right. \\
\times \exp [- & \left.\left.\left(\sum_{j \in\{-1,1\}} q_{j}+\sum_{i} \lambda \sum_{j \neq 0} \tilde{\sigma}_{i j}\left(k_{w}^{(r)}, z, \theta\right)\right) \tau_{w}^{(r)}\right]\right\} \\
& \times \exp \left[-\left(\sum_{j \in\{-1,1\}} q_{j}+\sum_{i} \lambda \sum_{j \neq 0} \tilde{\sigma}_{i j}\left(k_{W+1}^{(r)}, z, \theta\right)\right)\left(1-t_{W}^{(r)}\right)\right],
\end{aligned}
$$

where we use the same simulation draws as in Step 1, but replace each $\tilde{p}_{i j}$ with $\tilde{\sigma}_{i j}$, which is a function of $\theta$. The second stage estimates are then given by

$$
\check{\theta}=\arg \max _{\theta} \sum_{m=1}^{M} \sum_{z} P_{m}(z) \sum_{n=1}^{N} \ln \check{L}_{m n}(\theta ; \tilde{q}, \tilde{\alpha}, z) .
$$




\subsection{Results}

The structural parameter estimates for chain stores are presented in Table 3 . In the first set of columns we present the results where all entry and exit decisions are aggregated over the year. In the second set we use the information on the exact date of Wal-Mart entry, while the third set removes the controls for unobserved heterogeneity. In all cases, we calculate standard errors using the approach of Ackerberg, Chen, and Hahn (2012).

All three sets of estimates show Wal-Mart having substantial effects on chain flow profits that dwarf the effects of other chain and fringe stores. Despite these large effects, the estimates aggregating over Wal-Mart entry times (column 1) and using the exact date of Wal-Mart entry (column 2) show virtually identical parameter estimates, suggesting that, in this case, integrating out over entry times does not contaminate our estimates.

The effects of competition on chain profits, however, are substantially lower in the third set of columns that do not control for unobserved heterogeneity. The coefficients on the number of Wal-Marts and number of fringe stores are over thirty percent higher in columns 1 and 2 than in column 3, with the effect of the other chain stores almost four times as high. This is to be expected since, all else equal, higher unobserved demand will be correlated with more entry leading to estimates of competition that are biased downward. Controlling for such bias is important for our counterfactual analysis since the degree to which different firm types face differential competitive pressures from each type of rival will determine who thrives and who fails as the market evolves.

Markets with higher values of the unobserved state face lower building costs for chain firms and lower diminishing returns for increasing chain size, but the costs of entering the market are higher. Other coefficients are as expected-population increases profits and the costs of building stores is substantial, with even higher costs incurred for entering a market.

Results for the three specifications for fringe firms are presented in Table 4. We again see negative effects of Wal-Mart on fringe profits in all three specifications with the timeaggregated results (column 1) very close to those that conditional on Wal-Mart entry times (column 2). In contrast to the chain stores, Wal-Mart's effects on fringe stores are smaller when we account for unobserved heterogeneity. An additional chain store negatively affects profits of fringe stores, with the effect being about that half that of an additional Wal-Mart. By comparison, the effect of an additional chain store from a competing chain was a little over one-sixth of the effect of an additional Warmart for chain profits. Hence, Wal-Mart appears to have a greater relative effect on chain stores than fringe stores. Moreover, the impact of competition from both types of rivals is smaller for the fringe than for the chains, suggesting that fringe stores are indeed more differentiated in product space than their chain rivals. Since differentiation in this industry mainly involves focusing on more narrow segments of the consumer base (e.g. ethnic foods, organic meats and produce) it makes 
sense that the benefits of scale would be more muted here, creating an opening for smaller scale firms to counter the cost advantage enjoyed by the larger chains.

Due perhaps to the importance of distribution networks (which rely on achieving a minimal local scale), having more fringe competitors raises profits at first, with competitive effects dominating as the number of fringe competitors increases. This positive spillover is robust to the inclusion of correlated unobservables. Population again has a positive effect on profits and there are significant store building costs. Similar to chain stores, higher values of the unobserved state lower store building costs and lessen the competitive impact from fringe competitors. However, this latter effect is smaller for fringe stores.

\subsection{Counterfactuals}

The focus of our empirical analysis is on the differential impact of Wal-Mart on the chain and fringe segments. While Wal-Mart's earlier impact on the discount sector was unambiguously detrimental to small scale rivals, the impact on the grocery industry is much less clear. To evaluate the impact of Wal-Mart's entry on the market structure of the grocery industry, we conducted counterfactual experiments for each of the 205 markets that did not have a WalMart outlet in the beginning of our sample. In particular, we computed equilibrium policy functions for a counterfactual scenario in which Wal-Mart does not exist, ${ }^{21}$ and compared the temporal evolution of these markets under this counterfactual to the evolution implied by the estimated first stage policy functions recovered from the true data. ${ }^{22}$ Using these two sets of policy functions (true and counterfactual) we then simulated 10,000 future histories from the first period in the data (1994), and averaged over them (at the market level) to characterize differences in long-run equilibrium outcomes.

Table 5 illustrates the long-run impact of Wal-Mart's entry. The two panels of Table 5 contain the simulated equilibrium outcomes at year 20 (which corresponds to 2014 in calendar time) and includes several measures of market structure, including the average number of chain firms, chain stores, fringe stores, Wal-Mart stores, the average market shares of each of these three player types, the share (by store count) of the largest (C1) and three largest (C3) firms, and the Herfindahl-Hirschman Index (HHI) computed using the share of total square feet of selling space. ${ }^{23}$ Column 1 contains the average initial market popu-

\footnotetext{
${ }^{21}$ There is no guarantee that our counterfactual policy functions are unique. However, working in continuous time eliminates one sources of multiple equilibria (simultaneity) as well as being less-computationally intensive, implying we can search for other equilibria. Using 10,000 different draws on the starting values for the value functions and then iterating to a fixed point showed no evidence of multiple equilibria.

${ }^{22}$ Recall that we did not attempt to recover the full set of Wal-Mart's structural parameters because WalMart did not exit any markets over this period. The ability to make such a comparison is a benefit of using a two-step estimation approach rather than a (infeasible with this data structure) full-solution estimation approach.

${ }^{23}$ Our square footage calculation assumes that all Wal-Mart stores are 62,200 square feet, all chain stores
} 
lation, while column 2 contains the number of markets in a given category. The first row of each panel averages across all 205 markets, while the next four rows in each panel break this average out by census region.

Several clear patterns emerge. First, Wal-Mart has a sizeable negative impact on both the number of chain firms and number of chain stores that are active in a given market. Looking across regions, we find that the impact is fairly consistent across the Midwest, Northeast and South, but only about half as large in the West. This is consistent with both the higher level of political resistance that Wal-Mart has faced in these markets, and the fact that the more tightly clustered population centers in these markets are less suited to Wal-Mart's more diffuse outlet structure.

Second, Wal-Mart's presence actually leads to an expansion of the fringe. Note that this is in sharp contrast to what occurred in the discount store industry, where the small "mom and pop" stores retreated in the face of Wal-Mart's expansion. There are at least two likely reasons for this contrast. First, unlike the earlier experience of rural discount (general merchandise) stores, Wal-Mart faced a large number of well-established chain stores in the supermarket industry that had already made similar investments in scale and IT and were providing a range of products that overlapped very closely with Wal-Mart's offerings. Second, and consistent with this overlap, as captured in the first stage policy function estimates, Wal-Mart competes more directly with chain firms than those in the fringe. As noted above, firms in the fringe are much more likely to be horizontally differentiated into a distinct local niche (such as providing an ethnic or gourmet focus) than the full-service chains. As Wal-Mart displaces the chains, this likely provides an even greater opportunity for differentiation by the fringe segment, while Wal-Mart's huge cost advantage (reflected in its large competitive impact on chain flow profits) represents a direct challenge to the survival of competing chains. The next set of results will further explore these points.

Before doing so, we note that the overall (and regional) impact of Wal-Mart actually leads to a decrease in market concentration along all three measures (C1, C3 and HHI). This is driven by the displacement of the chains by firms from the fringe. Even though Wal-Mart eventually becomes a large player in many of these markets, the decrease in the number of chain firms is significantly smaller than the decrease in the number of chain stores, leading to a more uniform market structure when Wal-Mart is present. While we do not have information on prices, under most models of retail competition a more uniform structure would yield tougher price competition amongst the remaining firms, likely leading to benefits for consumers.

Table 6 cuts the counterfactual results along several additional dimensions. The first

are 35,500 square feet and all fringe stores are 13,500 feet, which correspond to the empirical averages from the data. 
two columns contain the number of markets in each category and the average number of Wal-Mart's predicted to enter under the "actual" scenario. The remaining columns report the percentage change in the various market structure measures employed in Table 5. The first panel breaks the results out by census region (as in Table 5), illustrating the importance of accounting for unobserved heterogeneity in capturing regional variation (we will return to this point shortly). The next panel breaks the results out by market size. We label markets as either "small" or "large" based on whether their initial population is below or above the median value. Here we see a sharp contrast: while the negative impact on chains is the same in both small and large markets, the positive impact on the fringe is much stronger in the larger markets. This is consistent with the notion that the fringe is exploiting a greater opportunity for horizontal differentiation, as these opportunities would naturally increase with market size (i.e., a larger population yields more sub-markets large enough to support one or more stores tailored to more unique tastes). Similarly, we divide markets by "Slow", "Moderate", or "Fast" growth rates based on whether they are in the lower, middle, or upper tercile of the population growth distribution.

The last panel breaks markets out by their "unobserved type" as assigned by the finite mixture model employed above. ${ }^{24}$ While there were five points of support in the estimation, no markets were pre-dominantly assigned to the highest type. Higher values of the unobserved type are clearly associated with greater Wal-Mart entry and higher initial population levels. Viewed geographically (results not shown), markets with low values of the unobserved state tend to be in the western and mountain states as well as New England, while markets with high values of the unobserved state tend to be in the south and southern Atlantic states. Again, this is consistent with Wal-Mart's center of regional strength and areas where opposition to their expansion is weakest. The highest values of the unobserved state are also associated with a greater initial fringe presence.

Strikingly, in markets assigned the lowest value for the unobserved type, Wal-Mart has a strong negative impact on both chain and fringe stores. As a result, concentration actually increases with Wal-Mart's presence, as it moves towards being the dominant firm in the market. Note that this outcome most closely matches what happened in the discount industry. However, in the case of supermarket industry, it represents only a very small fraction of the overall set of markets (9 out of 205). Geographically, these are small, rural markets that initially had few chains and were essentially dominated by fringe players. Wal-Mart pushes both types of firms out and actually increases the equilibrium level of concentration. It seems likely that these markets are too small to support a diverse range of offerings and Wal-Mart's scale advantage is dominant.

\footnotetext{
${ }^{24}$ The probability $P_{m}(z)$ that market $m$ is of unobserved type $z$ is defined in (21). We take the mean state for a market to be $\bar{z}_{m}=\sum_{z} z P_{m}(z)$ and round $\bar{z}_{m}$ to the nearest mass point to label cities, respectively, as "More Negative", "Negative", "Zero", "Positive", or "More Positive".
} 
However, this is far from the modal outcome across the whole industry. For all other values of the unobserved state, the average impact on the fringe is positive and sharply increasing with the value of the unobserved state. The overall structure becomes less concentrated with Wal-Mart's presence. This is due to both the contraction of the chain segment, which is hurt more the greater the value of the unobserved state, and the fact that the fringe competes less directly with (i.e. is more differentiated from) Wal-Mart.

Table 7 considers the impact of ignoring heterogeneity, and presents the same sets of conditional means employed in Table 6, but using parameters (and counterfactual computations) estimated without accounting for unobserved heterogeneity. Perhaps not surprisingly, these results miss the rich heterogeneity apparent in Table 6: the regional variation is muted and the asymmetric impact in large and small markets vanishes. The last panel breaks things using the values of the unobserved state recovered earlier (which are ignored in this estimation). Note that we now lose the traditional story altogether, as the small set of rural "mom and pop" markets are now averaged in with the rest. Most importantly, the offsetting impact of Wal-Mart's expansion, namely its complementary relationship with the fringe, is sharply reduced as we no longer capture its competitive separation from Wal-Mart and the interplay with market size.

Table 8 explores the temporal evolution of market structure, providing some insight into why the expansion of the fringe did not show up in earlier studies and illustrating the importance of a dynamic structural model in this context. Focusing on the small market versus large market comparison, the table shows the equilibrium market structure in years $5,10,15$ and 20. As in year 20, the expansion of the fringe is most pronounced in earlier years in the larger markets where the scope for differentiation is greatest. However, across both sizes, the expansion of the fringe evolves more slowly than the contraction of the chains, reflecting the subtle impact of dynamics. Recall that Wal-Mart's direct impact on the flow profits of the fringe is negative, but the overall impact is positive since WalMart pushes out the chains, who compete more directly with them, shifting the competitive landscape toward the smaller scale competitors. Interestingly, Wal-Mart has recently started to shift its focus toward offering much smaller stores (e.g. Wal-Mart Neighborhood Markets and Wal-Mart Express, which are closer in size to corner grocers and convenience stores), perhaps acknowledging the importance of these more localized offerings and the decreasing role of local scale.

\section{Conclusion}

While recently developed two-step estimation methods have made it possible to estimate

large-scale dynamic games, performing simulations for counterfactual work or generating 
data remains severely constrained by the computational burden that arises due to simultaneous moves. We recast the standard discrete-time, simultaneous-move game as a sequentialmove game in continuous time. This significantly reduces the computational cost, greatly expanding the breadth and applicability of these structural methods and making even fullsolution estimation feasible for very large games.

By building on an underlying discrete-choice random utility framework, our model preserves many of the desirable features of discrete-time models. In particular, we show that the insights from two-step estimation methods can be applied directly in our framework, resulting in another order of magnitude reduction in computational costs during estimation. We also show how to extend the model to accommodate incomplete sampling schemes, including time-aggregated data. Both are likely to be relevant for real-world datasets.

Using this formulation of a dynamic game in continuous time, we develop a dynamic model of retail competition that allows for substantial heterogeneity (both observed and unobserved) across agents and markets. We use the model to study the impact of WalMart's entry into the retail grocery industry on market structure. The results imply that Wal-Mart's entry on market structure varies greatly across markets, leading to an increase in market concentration in some markets which were initially served primarily by smaller fringe stores and to a sharp decrease in concentration in the majority of markets that were characterized by the presence of a number of large, dominant chains.

The inclusion of unobserved heterogeneity in the model is essential for uncovering these qualitatively distinct economic implications of Wal-Mart's entry across markets. Taken as a whole, the results of our analysis demonstrate the importance of incorporating substantial heterogeneity both across markets and firm types in estimating dynamic games of retail entry and competition, thereby highlighting the advantage of computationally light approaches for estimating and solving dynamic models with large state spaces.

\section{A Additional Details on Other Approaches to Estimation}

\section{A.1 Nested Pseudo-Likelihood Estimation}

In the main text we focus on the case of two-step pseudo maximum likelihood (PML) estimation where the hazards are estimated in a first step and used to form a pseudo-likelihood function which is in turn used to estimate the structural parameters. This is similar to the approach of Hotz and Miller (1993) and the pseudo maximum likelihood PML estimator of Aguirregabiria and Mira (2007). This process can be iterated to improve the estimates as in the nested pseudo likelihood (NPL) estimator, also proposed by Aguirregabiria and Mira (2007), in which the estimated structural parameters are used to obtain new hazards which are in turn used to obtain refined structural parameter estimates and so on. 
Let $\hat{h}^{0}$ denote an initial consistent estimator of the vector of hazards $h$ define in (16). Then let $\hat{\theta}^{1}$ denote the initial consistent two-step estimates of the structural parameters based on $\hat{h}^{0}$. We can iterate this process by using $\hat{\theta}^{1}$ to obtain new choice probabilities and hazards using the probability mapping $\Lambda$ as in $\hat{h}^{1}=\Lambda\left(\hat{\theta}^{1}, \hat{h}^{0}\right)$. We can therefore define the $K$-stage NPL estimator recursively as

$$
\hat{\theta}^{K}=\arg \max _{\theta \in \Theta} L_{M}\left(\theta, \hat{h}^{K-1}\right)
$$

where the hazards are in turn defined recursively as

$$
\hat{h}^{K}=\Lambda\left(\hat{\theta}^{K}, \hat{h}^{K-1}\right)
$$

\section{A.2 Forward-Simulation-Based Estimators}

Forward simulation methods like those of Hotz, Miller, Sanders, and Smith (1994) and Bajari, Benkard, and Levin (2007) can also be used in the continuous time framework we have developed. We take as given that the reduced form hazard of firm $i$ choosing action $j$ in state $k, h_{i j k}$, has been estimated in a first stage and that the choice-specific shocks follow a type I extreme value distribution.

In this case, $h_{i j k}$ is given by:

$$
\begin{aligned}
h_{i j k} & =\lambda \sigma_{i j k} \\
& =\frac{\lambda \exp \left(V_{i j k}-\psi_{i j k}\right)}{\sum_{j^{\prime}} \exp \left(V_{i j^{\prime} k}-\psi_{i j^{\prime} k}\right)}
\end{aligned}
$$

implying that the log differences in the hazards between action $j$ and 1 can be expressed as:

$$
\ln \left(h_{i j k}\right)-\ln \left(h_{i 1 k}\right)=V_{i j k}-V_{i 1 k}-\psi_{i j k}+\psi_{i 1 k}
$$

We assume the instantaneous payoff to beginning in state $k$ can be expressed as:

$$
u_{i k}=X_{i}(k) \alpha
$$

where $X_{i}(k)$ a row vector of dimension $\bar{X} \leq K$ associated with state $k$ and $\alpha$ is a $\bar{X} \times 1$ vector of coefficients to be estimated. Note that this is without loss of generality as $\bar{X}$ could equal the number of states. Again without loss of generality, we express the cost of changing the state as:

$$
\psi_{i j k}=Z_{i}(j, k) \psi
$$

where $Z_{i}(j, k)$ is a row vector of dimension $\bar{Z} \leq K$ associated with changing the state using 
action $j$ in state $k$. Our forward simulation methods will then collect values for the row vectors above.

Let $\hat{\delta}_{i}$ denote the estimated strategy or policy function of firm $i$ associated with the estimated hazards $\hat{h}$. Given values for the structural parameters, $\theta$, the ex-ante value function implied by these policies is

$$
\bar{V}_{i k}(\hat{\delta}, \hat{h}, \theta)=\mathrm{E}\left[\int_{0}^{\infty} \mathrm{e}^{-\rho t} u_{k_{t}} d t \mid k_{0}=k\right]
$$

where the expectation is taken with respect to the continuous time joint stochastic process $\left(k_{t}, \varepsilon_{t}\right)$ over the infinite time horizon given the estimated parameters $\hat{h}$ and strategy profile $\hat{\delta}$.

We can now approximate $V_{i j k}$ and $V_{i 1 k}$ using forward simulation methods. Specifically, for each of $R$ paths given initial state $k *$ and choice $j *$, we initialize the $1 \times \bar{X}$ vector $X_{i j^{*} r}$, the $1 \times \bar{Z}$ vector $Z_{i j^{*} r}$, and the scalar $\epsilon_{i j^{*} r}$ to zero. We then:

1. Draw the time of the next move arrival, $\tau(1, r)$ where the rate is given by the sum of the arrival processes associated with each of the players and nature (the $\lambda$ 's and $q$ 's).

2. Conditional on a move arrival, use the arrival processes to draw which player, or alternatively nature, received the move arrival.

3. Draw an action using the estimates of the $\sigma_{i j k}$ 's if a player has moved or a state change using the estimates of the $q$ 's if nature has moved. The action or state change then results in a new state $k(1, r)$.

4. Add $X_{i}\left(k^{*}\right) \int_{0}^{\tau(1, r)} e^{-\rho t} d t$ to $X_{i j^{*} r}^{*}$. This gives the weight associated with the payoff while in state $k^{*}$ over the interval zero to $\tau(1, r)$.

5. If state $k(1, r)$ resulted from player $i$ taking action $j$, then:

(a) add $e^{-\rho \tau(1, r)} \ln \left(\sigma_{i j k}\right)$, the discounted expected value of $\epsilon_{i j k}$ that resulted in the state change, to $\epsilon_{j^{*}}^{*}$. This gives the expected adjustment from the unobservable preferences from action $j$ being optimal.

(b) add $e^{-\rho \tau(1, r)} Z_{i}\left(j, k^{*}\right)$ to $Z_{i j^{*} r}^{*}$. This gives the weight associated with the cost of changing the state through action $j$ in state $k^{*}$.

6. Iterate on the previous steps, where for the $m$ th move arrival replace $\tau(1, r)$ with $\tau(m, r), k^{*}$ with $k(m, r)$, and $k(1)$ with $k(m+1, r)$, until the the last move arrival is sufficiently far into the future that future weights are sufficiently small. 
The log differences in the hazards between action $j$ and 1 can then be expressed as:

$$
\begin{aligned}
\ln \left(h_{i j k}\right)-\ln \left(h_{i 1 k}\right)= & {\left[\sum_{r}\left(X_{i j r}^{*}-X_{i 1 r}^{*}\right) / R\right] \alpha+\left[\sum_{r}\left(\epsilon_{i j}^{*}-\epsilon_{i 1}^{*}\right) / R\right] } \\
& -\left[\sum_{r}\left(Z_{i j r}^{*}-Z_{i 1 r}^{*}\right) / R+Z_{i}(j, k)-Z_{i}(1, k)\right] \psi
\end{aligned}
$$

Note that all the terms in brackets can be calculated given the reduced form hazards implying that stacking the differences in hazards results in a linear system.

\section{A.2.1 Second Step Estimation for Hotz, Miller, Sanders, and Smith (1994)}

Following Hotz, Miller, Sanders, and Smith (1994), use (25) to form moment conditions. We then stack the moment conditions for each state and choose $\{\alpha, \psi\}$ it minimize the squared differences of (25) where the left-hand side has been replaced by the estimated hazards.

\section{A.2.2 Second Step Estimation for Bajari, Benkard, and Levin (2007)}

With the first-step estimated hazards $\hat{h}$ in hand and the ability to approximate the value function for any arbitrary collection of policies (included the estimated ones), estimation of the second step structural parameters can proceed as in Bajari, Benkard, and Levin (2007). They use the principle of revealed preference to construct a minimum distance objective function for the structural parameters based on the equilibrium conditions. In an MPE, no single firm should be able to improve their presented discounted profits by deviating from the estimated policy rule. We can estimate the value function for any firm $i$ in any state $k$ by using the estimated hazards to simulate equilibrium behavior. We can do the same for an alternative policy function that yields different hazards and then bulid an objective function based on minimizing violations of the equilibrium conditions.

The structural parameters $\theta$ can be estimated as usual by treating the estimated policies $\hat{\delta}=\left(\hat{\delta}_{1}, \ldots, \hat{\delta}_{N}\right)$ as the true policies in the equilibrium conditions,

$$
\bar{V}_{i}\left(s ; \hat{\delta}_{i}, \hat{\delta}_{-i}, \hat{h}, \theta\right) \geq \bar{V}_{i}\left(s ; \delta_{i}^{\prime}, \hat{\delta}_{-i}, \hat{h}, \theta\right) \quad \forall\left(i, k, \delta_{i}^{\prime}\right),
$$

and using them to form a minimum distance objective function

$$
S(\theta) \equiv \int\left[\min \left\{\bar{V}_{i}\left(k ; \hat{\delta}_{i}, \hat{\delta}_{-i}, \hat{h} ; \theta\right)-\bar{V}_{i}\left(k ; \delta_{i}^{\prime}, \hat{\delta}_{-i}, \hat{h} ; \theta\right), 0\right\}\right]^{2} d H\left(i, k, \delta_{i}^{\prime}\right)
$$

where $H$ is a distribution over the set of possible "inequalities" represented by tuples of the form $\left(i, k, \delta_{i}^{\prime}\right)$. By minimizing this function, we minimize the sum of squared violations of the equilibrium conditions, motivated by revealed preference. Under the true parameters 
$\theta$, the true policy should always yield higher discounted future payoffs than any alternative policy $\delta_{i}^{\prime}$ for each firm $i$.

\section{B Proofs}

\section{B.1 Proof of Proposition 1}

Let $\Sigma(\sigma)$ denote the $K \times K$ state transition matrix induced by the choice probabilities $\sigma$ and the continuation state function $l(\cdot, \cdot)$. Let $\tilde{Q}_{0}$ denote the matrix formed by replacing the diagonal elements of $Q_{0}$ with zeros. Finally, let $E(\sigma)$ be the $K \times 1$ matrix containing the ex-ante expected value of the immediate payoff (both the instantaneous payoff and the choice-specific shock) as defined in Proposition 1.

We can rewrite the value function in (1) in matrix form as

$$
\left[(\rho+\lambda) I-\left(Q_{0}-\tilde{Q}_{0}\right)\right] V(\sigma)=u+\tilde{Q}_{0} V(\sigma)+\lambda[\Sigma(\sigma) V(\sigma)+E(\sigma)] .
$$

Collecting terms involving $V(\sigma)$ yields

$$
\left[(\rho+\lambda) I-\lambda \Sigma(\sigma)-Q_{0}\right] V(\sigma)=u+\lambda E(\sigma)
$$

The matrix on the left hand side is strictly diagonally dominant since the diagonal of $Q$ equals the off-diagonal row sums, the elements of $\Sigma(\sigma)$ are in $[0,1]$, and $\rho>0$ by Assumption 1. Therefore, by the Levy-Desplanques theorem, this matrix is nonsingular (Horn and Johnson, 1985, Theorem 6.1.10). The result follows by multiplying both sides of the above equation by $\left[(\rho+\lambda) I-\lambda \Sigma(\sigma)-Q_{0}\right]^{-1}$.

\section{B.2 Proof of Proposition 2}

Let $v_{j k}=\psi_{j k}+V_{l(j, k)}$ denote the choice-specific value function, net of the choice-specific shock, for choice $j$ in state $k$. Given the additively separable structure of instantaneous payoffs, we can express the conditional choice probabilities in terms of the social surplus function of McFadden (1981):

$$
S\left(v_{0 k}, \ldots, v_{J-1, k} ; k\right) \equiv \operatorname{E}_{j \in \mathcal{A}}\left\{v_{j k}+\varepsilon_{j}\right\}
$$

This function depends implicitly on the joint distribution of $\varepsilon$. Under Assumption 2, by the Williams-Daly-Zachary theorem (Rust, 1994, Theorem 3.1), the function $S$ exists, $S$ is additive in the sense that for any $\alpha \in \mathbb{R}$,

$$
S\left(v_{0 k}+\alpha, \ldots, v_{J-1, k}+\alpha ; k\right)=S\left(v_{0 k}, \ldots, v_{J-1, k} ; k\right)+\alpha
$$


and the vector of CCPs equals the gradient of $S: \sigma_{k}=\nabla S\left(v_{0 k}, \ldots, v_{J-1, k} ; k\right)$. Let $\tilde{v}_{k}=$ $\left(v_{0 k}-v_{j^{\prime}, k}, \ldots, v_{J-1, k}-v_{j^{\prime}, k}\right)$ denote the $J-1$ vector of differenced choice-specific value functions, relative to choice $j^{\prime}$, with the $j^{\prime}$-th component omitted.

It follows from Proposition 1 of Hotz and Miller (1993) (also see Lemma 3.1 of Rust (1994)) that there is a one-to-one function $H$ which maps the differenced choice-specific value functions $\tilde{v}_{k}$ in $\mathbb{R}^{J-1}$ to choice probabilities $\sigma_{k}$ in $\Delta^{J}$, the $J$-dimensional unit simplex in $\mathbb{R}^{J}$. The result follows by noting that the $j$-th component of the inverse mapping yields $\tilde{v}_{j k}=\psi_{j k}-\psi_{j^{\prime} k}+V_{l(j, k)}-V_{l\left(j^{\prime}, k\right)}$ as a function of $j, j^{\prime}$, and $\sigma_{k}$.

\section{B.3 Proof of Proposition 3}

Recall from the proof of Proposition 2 that, by the Williams-Daly-Zachary theorem (Rust, 1994, Theorem 3.1) and Proposition 1 of Hotz and Miller (1993), we have $\tilde{v}_{k}=H^{-1}\left(\sigma_{k}\right)$. Then, by the aforementioned additivity property of $S$,

$$
\begin{aligned}
\mathrm{E} \max _{j}\left\{v_{j k}+\varepsilon_{j}\right\} & =\mathrm{E} \max _{j}\left\{v_{j k}-v_{j^{\prime} k}+\varepsilon_{j}-\varepsilon_{j^{\prime}}\right\}+v_{j^{\prime} k}+\mathrm{E} \varepsilon_{j^{\prime}} \\
& =S\left(v_{0 k}-v_{j^{\prime} k}, \ldots, v_{J-1, k}-v_{j^{\prime} k} ; k\right)+v_{j^{\prime} k}+\mathrm{E} \varepsilon_{j^{\prime}} \\
& =V_{l\left(j^{\prime}, k\right)}+\psi_{j^{\prime} k}+\Gamma^{2}\left(j^{\prime}, \sigma_{k}\right),
\end{aligned}
$$

where $\Gamma^{2}\left(j^{\prime}, \sigma_{k}\right)=S\left(H^{-1}\left(\sigma_{k}\right) ; k\right)+\mathrm{E} \varepsilon_{j^{\prime}}$.

\section{B.4 Proof of Proposition 4}

For simplicity, suppose that $j=0$ is a continuation action such that $l(0, k)=k .^{25}$ Let $\left(j_{k}^{1}, \ldots, j_{k}^{D_{k}}\right)$ denote a generic sequence of $D_{k}$ decisions by which state $k^{*}$ is attainable from state $k$. Similarly, let $l_{k}^{d}$ denote the intermediate state in which the $d$-th decision is made, where $l_{k}^{1}=k$ and $l_{k}^{d}=l\left(j_{k}^{d-1}, l_{k}^{d-1}\right)$. Then, by recursively applying Proposition 2 for the continuation choice $j=0$, we can write

$$
V_{k}=V_{k^{*}}+\sum_{d=1}^{D_{k}}\left(\psi_{j_{k}^{d}, l_{k}^{d}}-\psi_{0, l_{k}^{d}}\right)+\sum_{d=1}^{D_{k}} \Gamma^{1}\left(0, j_{k}^{d}, \sigma_{l_{k}^{d}}\right) .
$$

Applying a similar procedure for each $l \neq k$ for which $q_{k l}>0$ implies that we can write the differences $V_{l}-V_{k}$ on the right-hand side of (2) in terms of a difference of terms of the form in (26), where the $V_{k^{*}}$ term cancels leaving only sums of instantaneous payoffs $\psi_{j k}$ and functions of the CCPs $\sigma_{k}$. Finally, using Proposition 3 and additivity, we can express the remaining term $\lambda \operatorname{Emax}_{j}\left\{\psi_{j k}+\varepsilon_{j}+V_{l(j, k)}-V_{k}\right\}$ as $\lambda \Gamma^{2}\left(0, \sigma_{k}\right)+\lambda \psi_{0 k}$.

\footnotetext{
${ }^{25}$ Otherwise, we have to begin from some suitable state $k^{\prime}$ and choice $j^{\prime}$ such that $l\left(j^{\prime}, k^{\prime}\right)=k$ so that $V_{k}$ is on the left-hand side of (4).
} 


\section{B.5 Proof of Propositions 5 and 6}

Given a collection of equilibrium best response probabilities $\left\{\sigma_{i}\right\}_{i=1}^{N}$, we can obtain a matrix expression for the value function $V_{i}\left(\sigma_{i}\right)$ by rewriting (7). Let $\Sigma_{m}\left(\sigma_{m}\right)$ denote the $K \times K$ state transition matrix induced by the choice probabilities $\sigma_{m}$ and the continuation state function $l(m, \cdot, \cdot)$. Let $\tilde{Q}_{0}$ denote the matrix formed by replacing the diagonal elements of $Q_{0}$ with zeros. Finally, let $E_{i}\left(\sigma_{i}\right)$ be the $K \times 1$ matrix containing the ex-ante expected value of the immediate payoff (both the instantaneous payoff and the choice-specific shock) for player $i$ as defined in Proposition 6 .

Then, we can rewrite (7) in matrix form as

$$
\begin{aligned}
{\left[(\rho+N \lambda) I-\left(Q_{0}-\right.\right.} & \left.\left.\tilde{Q}_{0}\right)\right] V_{i}\left(\sigma_{i}\right) \\
& =u_{i}+\tilde{Q}_{0} V_{i}\left(\sigma_{i}\right)+\sum_{m \neq i} \lambda \Sigma_{m}\left(\sigma_{m}\right) V_{i}\left(\sigma_{i}\right)+\lambda\left[\Sigma_{i}\left(\sigma_{i}\right) V_{i}\left(\sigma_{i}\right)+E_{i}\left(\sigma_{i}\right)\right] .
\end{aligned}
$$

Collecting terms involving $V_{i}\left(\sigma_{i}\right)$ yields

$$
\left[(\rho+N \lambda) I-\sum_{m=1}^{N} \lambda \Sigma_{m}\left(\sigma_{m}\right)-Q_{0}\right] V_{i}\left(\sigma_{i}\right)=u_{i}+\lambda E_{i}\left(\sigma_{i}\right) .
$$

The matrix on the left hand side is strictly diagonally dominant since the diagonal of $Q$ equals the off-diagonal row sums, the elements of each matrix $\Sigma_{m}\left(\sigma_{m}\right)$ are in $[0,1]$ for all $m$, and $\rho>0$ by Assumption 1. Therefore, by the Levy-Desplanques theorem, this matrix is nonsingular (Horn and Johnson, 1985, Theorem 6.1.10). Hence,

$$
V_{i}\left(\sigma_{i}\right)=\left[(\rho+N \lambda) I-\sum_{m=1}^{N} \lambda \Sigma_{m}\left(\sigma_{m}\right)-Q_{0}\right]^{-1}\left[u_{i}+\lambda E_{i}\left(\sigma_{i}\right)\right]
$$

This representation is similar to the linear expression for the ex-ante value function in the discrete time model of Pesendorfer and Schmidt-Dengler (2007).

Now, define the mapping $\Upsilon:[0,1]^{N \times J \times K} \rightarrow[0,1]^{N \times J \times K}$ by stacking the best response probabilities. This mapping defines a fixed point problem for the equilibrium choice probabilities $\sigma_{i j k}$ as follows:

$$
\Upsilon_{i j k}(\sigma)=\int 1\left\{\varepsilon_{i j^{\prime}}-\varepsilon_{i j} \leq \psi_{i j k}-\psi_{i j^{\prime} k}+V_{i, l(i, j, k)}\left(\sigma_{i}\right)-V_{i, l\left(i, j^{\prime}, k\right)}\left(\sigma_{i}\right) \quad \forall j^{\prime} \in \mathcal{A}_{i}\right\} f\left(\varepsilon_{i}\right) d \varepsilon_{i} .
$$

The mapping $\Upsilon$ is a continuous function from a compact space onto itself. By Brouwer's theorem, it has a fixed point. The fixed point probabilities imply Markov strategies that constitute a Markov perfect equilibrium. 


\section{B.6 Proof of Proposition 7}

Step 1: Uniform Convergence of $L_{M}$ to $L$-We apply the uniform law of large numbers of Newey and McFadden (1994, Lemma 2.4) to establish uniform convergence. The data are independent and identically distributed under Assumption 3, the parameter space is compact under Assumption 5, the observation likelihood is continuous at each $(\theta, h)$ with probability one, and the observation likelihood is strictly bounded between 0 and 1 under additive separability of the idiosyncratic shocks and Assumption 2, and since the rates $\lambda$ and $q_{k l}$ are bounded for all $k$ and $l$.

Step 2: Consistency of $\hat{\theta}$ - By assumption, $\hat{h}$ is a $\sqrt{M}$-consistent M-estimator. Let $R_{M}(h)=\frac{1}{M} \sum_{m=1}^{M} r_{m}(h)$ denote it's objective function and let $r_{m} \equiv r_{m}\left(h^{0}\right)$. (For example, for nonparametric MLE we would have $\left.r_{m}(h)=\sum_{n=2}^{T} \ln P_{k_{m, n-1}, k_{m n}}(\Delta ; h)\right)$. We have shown above that $L_{M}$ converges uniformly in probability to $L$. $L$ is also uniformly continuous in $\theta$ and $h$. Therefore, by Lemma 24.1 of Gourieroux and Monfort (1995), $L_{M}(\theta, \hat{h})$ converges in probability to $L\left(\theta, h^{0}\right)$ uniformly in $\theta$. Then, Assumption 6 implies that $\theta^{0}$ is the only element of $\Theta$ for which $h^{0}=\Lambda\left(\theta, h^{0}\right)$. This assumption plays a role similar to the conditional density identification condition in conditional maximum likelihood. By the Kullback-Leibler information inequality, it follows that $\theta^{0}$ is the unique maximizer of $L\left(\theta, h^{0}\right)$ in $\Theta$. It follows by Theorem 2.1 of Newey and McFadden (1994) that $\hat{\theta}=\arg \max _{\theta \in \Theta} L_{M}(\theta, \hat{h}) \stackrel{\mathrm{p}}{\rightarrow} \theta^{0}$.

Step 3: Asymptotic Normality of $\hat{\theta}$ - The first order conditions for $\hat{\theta}$ are $\nabla_{\theta} L_{M}(\hat{\theta}, \hat{h})=0$. By a mean value expansion between $\left(\theta^{0}, h^{0}\right)$ and $(\hat{\theta}, \hat{h})$ and by consistency of the latter,

$$
0=\nabla_{\theta} L_{M}\left(\theta^{0}, h^{0}\right)+\nabla_{\theta \theta^{\top}} L_{M}\left(\theta, h^{0}\right)\left(\hat{\theta}-\theta^{0}\right)+\nabla_{\theta h^{\top}} L_{M}\left(\theta^{0}, h^{0}\right)\left(\hat{h}-h^{0}\right)+o_{p}(1) .
$$

By the central limit theorem and information matrix equality, $\nabla_{\theta \theta^{\top}} L_{M}\left(\theta^{0}, h^{0}\right) \stackrel{\mathrm{p}}{\rightarrow}-\Omega_{\theta \theta^{\top}}$ and $\nabla_{\theta h^{\top}} L_{M}\left(\theta^{0}, h^{0}\right) \stackrel{\mathrm{p}}{\rightarrow}-\Omega_{\theta h^{\top}}$. It follows that

$$
\sqrt{M}\left(\hat{\theta}-\theta^{0}\right)=\Omega_{\theta \theta^{\top}}^{-1}\left\{\Omega_{\theta h^{\top}}\left(\frac{1}{\sqrt{M}} \sum_{m=1}^{M} \nabla_{h} r_{m}\right)+\left(\frac{1}{\sqrt{M}} \sum_{m=1}^{M} \nabla_{\theta} s_{m}\right)\right\}+o_{p}(1) .
$$

Finally, we note that under the assumptions $\Lambda$ is continuously differentiable and the transition probabilities are bounded away from zero. The regularity conditions of Theorem 5.1 of Newey and McFadden (1994) are satisfied, so the generalized information matrix equality holds (Newey and McFadden, 1994, p. 2163), $\mathrm{E}\left[\nabla_{h} r_{m} \nabla_{\theta^{\top}} s_{m}\right]=0$, and $\mathrm{E}\left[\nabla_{h} r_{m} \nabla_{h^{\top}} s_{m}\right]=$ I. Thus,

$$
\frac{1}{\sqrt{M}}\left(\sum_{m=1}^{M} \nabla_{\theta} s_{m}\right)-\Omega_{\theta h^{\top}}\left(\frac{1}{\sqrt{M}} \sum_{m=1}^{M} \nabla_{h} r_{m}\right) \stackrel{\mathrm{d}}{\rightarrow} \mathrm{N}\left(0, \Omega_{\theta \theta^{\top}}+\Omega_{\theta h^{\top}} \Sigma \Omega_{\theta h^{\top}}^{\top}\right) .
$$


The result holds by applying the continuous mapping theorem.

\section{References}

Ackerberg, D., X. Chen, and J. Hahn (2012). A practical asymptotic variance estimator for two-step semiparametric estimators. Review of Economics and Statistics 94, 481-498.

Aguirregabiria, V. and P. Mira (2002). Swapping the nested fixed point algorithm: A class of estimators for discrete Markov decision models. Econometrica 70, 1519-1543.

Aguirregabiria, V. and P. Mira (2007). Sequential estimation of dynamic discrete games. Econometrica 75, 1-53.

Aguirregabiria, V. and P. Mira (2010). Dynamic discrete choice structural models: A survey. Journal of Econometrics 156, 38-67.

Altuğ, S. and R. A. Miller (1998). The effect of work experience on female wages and labour supply. Review of Economic Studies 65, 45-85.

Arcidiacono, P. and R. A. Miller (2011). Conditional choice probability estimation of dynamic discrete choice models with unobserved heterogeneity. Econometrica 79, 18231867.

Arcidiacono, P. and R. A. Miller (2014). Nonstationary dynamic models with finite dependence. Working paper, Duke University.

Arcidiacono, P., H. Sieg, and F. Sloan (2007). Living rationally under the volcano? An empirical analysis of heavy drinking and smoking. International Economic Review 48 , $37-65$.

Bajari, P., C. L. Benkard, and J. Levin (2007). Estimating dynamic models of imperfect competition. Econometrica 75, 1331-1370.

Bajari, P., H. Hong, and D. Nekipelov (2013). Game theory and econometrics: A survey of some recent research. In D. Acemoglu, M. Arellano, and E. Dekel (Eds.), Advances in Econometrics: Tenth World Congress, Volume 3, Chapter 1, pp. 3-52. Cambridge: Cambridge University Press.

Bajari, P., H. Hong, and S. P. Ryan (2007). Identification and estimation of discrete games of complete information. Econometrica 78, 1529-1568.

Basker, E. (2007). The causes and consequences of Wal-Mart's growth. Journal of Economic Perspectives 21, 177-198. 
Basker, E. and M. Noel (2009). The evolving food chain: Competitive effects of Wal-Mart's entry into the supermarket industry. Journal of Economics and Management Strategy 18, 977-1009.

Beauchamp, A. (2014). Regulation, imperfect competition, and the U.S. abortion market. International Economic Review.

Bellman, R. E. (1957). Dynamic Programming. Princeton, NJ: Princeton University Press.

Berry, S. T. (1992). Estimation of a model of entry in the airline industry. Econometrica 60, 889-917.

Billingsley, P. (1961). Statistical Inference for Markov Processes. Chicago: University of Chicago Press.

Blevins, J. R. (2013). Identifying restrictions for finite parameter continuous time models with discrete time data. Working Paper 13-01, Ohio State University.

Blevins, J. R. (2014). Identification and estimation of continuous time dynamic discrete choice games. Working paper, Ohio State University.

Bresnahan, T. F. and P. C. Reiss (1991). Empirical models of discrete games. Journal of Econometrics 48, 57-81.

Collard-Wexler, A. (2013). Demand fluctuations in the ready-mix concrete industry. Econometrica 81, 1003-1037.

Cosman, J. (2014). Industry dynamics and the value of variety in nightlife: Evidence from Chicago. Working paper, University of British Columbia.

Cuthbert, J. R. (1973). The logarithm function for finite-state Markov semi-groups. Journal of the London Mathematical Society s2-6, 524-532.

Doraszelski, U. and K. L. Judd (2012). Avoiding the curse of dimensionality in dynamic stochastic games. Quantitative Economics 3, 53-93.

Einav, L. (2010). Not all rivals look alike: Estimating an equilibrium model of the release date timing game. Economic Inquiry 48, 369-390.

Elfving, G. (1937). Zur theorie der Markoffschen ketten. Acta Societatis Scientiarum Fennica Nova Series A 2, 1-17.

Ellickson, P. B. and P. L. Grieco (2013). Wal-Mart and the geography of grocery retailing. Journal of Urban Economics 75, 1-14. 
Ellickson, P. B., S. Houghton, and C. Timmins (2010). Estimating network economies in retail chains: A revealed preference approach. Working Paper 15832, NBER.

Ericson, R. and A. Pakes (1995). Markov-perfect industry dynamics: A framework for empirical work. Review of Economics and Statistics 62, 53-82.

Gourieroux, C. and A. Monfort (1995). Statistics and Econometric Models, Volume 2. Cambridge University Press.

Haltiwanger, J., R. Jarmin, and C. Krizan (2010). Mom-and-pop meet big-box: Complements or substitutes? Journal of Urban Economics 67, 116-134.

Hansen, L. P. and T. J. Sargent (1983). The dimensionality of the aliasing problem in models with rational spectral densities. Econometrica 51, 377-387.

Holmes, T. (2011). The diffusion of Wal-Mart and economies of density. Econometrica 79, 253-302.

Hong, H., W. Li, and B. Wang (2012). Estimation of dynamic discrete models from time aggregated data. Working paper, Stanford University.

Horn, R. A. and C. R. Johnson (1985). Matrix Analysis. New York: Cambridge University Press.

Hotz, V. J. and R. A. Miller (1993). Conditional choice probabilities and the estimation of dynamic models. Review of Economic Studies 60, 497-529.

Hotz, V. J., R. A. Miller, S. Sanders, and J. Smith (1994). A simulation estimator for dynamic models of discrete choice. Review of Economic Studies 61, 265-289.

Igami, M. (2014). Estimating the innovator's dilemma: Structural analysis of creative destruction in the hard disk drive industry. Working paper, Yale University.

Jia, P. (2008). What happens when Wal-Mart comes to town: An empirical analysis of the discount retail industry. Econometrica 76, 1263-1316.

Johansen, S. (1974). Some results on the imbedding problem for finite Markov chains. Journal of the London Mathematical Society s2-8, 345-351.

Karlin, S. and H. M. Taylor (1975). A First Course in Stochastic Processes (Second ed.). San Diego, CA: Academic Press.

Keane, M. and K. I. Wolpin (1997). The career decisions of young men. Journal of Political Economy 105, 473-522. 
Kingman, J. F. C. (1962). The imbedding problem for finite Markov chains. Probability Theory and Related Fields 1, 14-24.

Maskin, E. and J. Tirole (2001). Markov perfect equilibrium: I. Observable actions. Journal of Economic Theory 100, 191-219.

Matsa, D. A. (2011). Competition and product quality in the supermarket industry. Quarterly Journal of Economics 126, 1539-1591.

Mazur, J. (2014). Can stricter bankruptcy laws discipline capital investment? Evidence from the U.S. airline industry. Working paper, Duke University.

McFadden, D. (1981). Econometric models of probabilistic choice. In C. F. Manski and D. McFadden (Eds.), Structural Analysis of Discrete Data. Cambridge, MA: MIT Press.

Milgrom, P. R. and R. J. Weber (1985). Distributional strategies for games with incomplete information. Mathematics of Operations Research 10, 619-632.

Moler, C. and C. V. Loan (1978). Nineteen dubious ways to compute the exponential of a matrix. SIAM Review 20, 801-836.

Nevskaya, Y. and P. Albuquerque (2012). A continuous time model of product usage: Measuring the effect of product design and rewards in online games. Working paper.

Newey, W. K. and D. McFadden (1994). Large sample estimation and hypothesis testing. In R. F. Engle and D. McFadden (Eds.), Handbook of Econometrics, Volume 4, Amsterdam. North Holland.

Pakes, A. and P. McGuire (1994). Computing Markov-perfect Nash equilibria: Numerical implications of a dynamic differentiated product model. The RAND Journal of Economics 25, 555-589.

Pakes, A., M. Ostrovsky, and S. Berry (2007). Simple estimators for the parameters of discrete dynamic games (with entry/exit examples). The RAND Journal of Economics 38, 373-399.

Pesendorfer, M. and P. Schmidt-Dengler (2007). Asymptotic least squares estimators for dynamic games. Review of Economic Studies 75, 901-928.

Phillips, P. C. (1973). The problem of identification in finite parameter continuous time models. Journal of Econometrics 1, 351-362.

Rust, J. (1987). Optimal replacement of GMC bus engines: An empirical model of Harold Zurcher. Econometrica 55, 999-1013. 
Rust, J. (1994). Estimation of dynamic structural models, problems and prospects: Discrete decision processes. In C. Sims (Ed.), Advances in Econometrics: Sixth World Congress, Volume 2, Chapter 4, pp. 119-170. Cambridge: Cambridge University Press.

Ryan, S. P. (2012). The costs of environmental regulation in a concentrated industry. Econometrica 80, 1019-1061.

Schiraldi, P., H. Smith, and Y. Takahasi (2012). Estimating a dynamic game of spatial competition: The case of the U.K. supermarket industry. Working paper.

Schmidt-Dengler, P. (2006). The timing of new technology adoption: The case of MRI. Working paper, London School of Economics.

Sidje, R. B. (1998). Expokit: A software package for computing matrix exponentials. ACM Transactions on Mathematical Software 24, 130-156.

Singer, B. and S. Spilerman (1976). The representation of social processes by Markov models. The American Journal of Sociology 82, 1-54.

Sweeting, A. (2013). Dynamic product positioning in differentiated product markets: The effect of fees for musical performance rights on the commercial radio industry. Econometrica 81, 2013.

Weintraub, G. Y., C. L. Benkard, and B. Van Roy (2008). Markov perfect industry dynamics with many firms. Econometrica 76, 1375-1411. 
Table 1: Summary Statistics

\begin{tabular}{|c|c|c|c|}
\hline & "Mean & "S.D. & Max. \\
\hline Number of Chains Present ${ }^{a}$ & 2.559 & 0.024 & 7 \\
\hline Average No. of Stores per Chain ${ }^{b}$ & 3.727 & 0.040 & 32 \\
\hline Number of Wal-Marts Present ${ }^{a}$ & 1.004 & 0.142 & 12 \\
\hline Number of Fringe Firms Present ${ }^{a}$ & 12.997 & 0.823 & 47 \\
\hline Number of New Chain Stores ${ }^{c}$ & 0.277 & 0.012 & 5 \\
\hline Number of Exiting Chain Stores & 0.224 & 0.011 & 7 \\
\hline Number of New Fringe Stores & 0.825 & 0.021 & 10 \\
\hline Number of Exiting Fringe Stores & 0.908 & 0.023 & 11 \\
\hline Number of New Wal-Marts & 0.177 & 0.008 & 3 \\
\hline Number of Exiting Wal-Marts & 0.002 & 0.001 & 1 \\
\hline Population Increase & 0.042 & 0.004 & 1 \\
\hline Population Decrease & 0.004 & 0.001 & 1 \\
\hline
\end{tabular}

Table 2: Response to Initial Wal-Mart Entry

\begin{tabular}{lccc}
\hline \hline & $\begin{array}{c}\text { Year } \\
\text { Before }\end{array}$ & $\begin{array}{c}\text { Year } \\
\text { During }\end{array}$ & $\begin{array}{c}\text { Year } \\
\text { After }\end{array}$ \\
\hline Number of New Chain Stores & 0.311 & 0.211 & 0.189 \\
& $(0.064)$ & $(0.054)$ & $(0.041)$ \\
Number of Exiting Chain Stores & 0.122 & 0.156 & 0.189 \\
& $(0.038)$ & $(0.044)$ & $(0.050)$ \\
Number of New Fringe Stores & 0.867 & 0.711 & 0.767 \\
& $(0.117)$ & $(0.105)$ & $(0.102)$ \\
Number of Exiting Fringe Stores & 0.789 & 0.844 & 0.833 \\
& $(0.114)$ & $(0.118)$ & $(0.132)$
\end{tabular}

Standard errors in parentheses. Based on 90 markets where Wal-Mart is first observed to enter. 
Table 3: Chain Firm Parameters

\begin{tabular}{lrrrrrrr}
\hline \hline & & \multicolumn{3}{c}{ With Wal-Mart } & \multicolumn{3}{c}{ No Unobserved } \\
& \multicolumn{2}{c}{ Time-aggregated } & \multicolumn{2}{c}{ Entry } & Times & \multicolumn{2}{c}{ Heterogeneity } \\
& Coeff. & S.E. & Coeff. & S.E. & Coeff. & S.E. \\
\hline Constant $\left(\theta_{0}^{\mathrm{c}}\right)$ & 4.470 & $(0.768)$ & 4.403 & $(0.749)$ & 2.561 & $(0.409)$ \\
Number of Chain Stores $\left(\theta_{1}^{\mathrm{c}}\right)$ & -0.065 & $(0.024)$ & -0.067 & $(0.024)$ & -0.017 & $(0.014)$ \\
Number of Wal-Marts $\left(\theta_{2}^{\mathrm{c}}\right)$ & -0.375 & $(0.148)$ & -0.383 & $(0.139)$ & -0.278 & $(0.108)$ \\
Number of Fringe Stores $\left(\theta_{3}^{\mathrm{c}}\right)$ & -0.052 & $(0.017)$ & -0.053 & $(0.017)$ & -0.040 & $(0.012)$ \\
Number of Own Stores $\left(\theta_{4}^{\mathrm{c}}\right)$ & -0.039 & $(0.081)$ & -0.044 & $(0.084)$ & 0.104 & $(0.051)$ \\
Number of Own Stores Sq. $/ 100\left(100 \times \theta_{5}^{\mathrm{c}}\right)$ & -0.182 & $(0.432)$ & -0.165 & $(0.445)$ & -0.265 & $(0.166)$ \\
Population $\left(\theta_{6}^{\mathrm{c}}\right)$ & 0.176 & $(0.114)$ & 0.213 & $(0.111)$ & 0.267 & $(0.075)$ \\
Unobserved State $\left(\theta_{7}^{\mathrm{c}}\right)$ & -0.956 & $(0.881)$ & -0.968 & $(0.806)$ & & \\
Unobserved State $\times$ Number of Own Stores $\left(\theta_{8}^{\mathrm{c}}\right)$ & 0.245 & $(0.199)$ & 0.249 & $(0.191)$ & & \\
& & & & & & \\
Entry Cost $\left(\eta_{0}^{\mathrm{c}}\right)$ & -18.377 & $(0.805)$ & -18.400 & $(0.807)$ & -17.643 & $(0.953)$ \\
Entry Cost $\times$ Unobserved State $\left(\eta_{1}^{\mathrm{c}}\right)$ & -5.151 & $(1.621)$ & -5.148 & $(1.676)$ & & \\
Store Building Cost $\left(\kappa_{0}^{\mathrm{c}}\right)$ & -5.068 & $(0.876)$ & -5.073 & $(0.870)$ & -4.494 & $(0.782)$ \\
Store Building Cost $\times$ Unobserved State $\left(\kappa_{1}^{\mathrm{c}}\right)$ & 3.513 & $(0.968)$ & 3.508 & $(0.986)$ & & \\
Exit Value $\left(\phi_{0}^{\mathrm{c}}\right)$ & 15.913 & $(0.888)$ & 15.912 & $(0.896)$ & 15.044 & $(0.633)$ \\
Exit Value $\times$ Unobserved State $\left(\phi_{1}^{\mathrm{c}}\right)$ & 4.166 & $(1.261)$ & 4.126 & $(1.274)$ & & \\
\hline
\end{tabular}


Table 4: Fringe Firm Parameters

\begin{tabular}{lrrrrrr}
\hline \hline & & \multicolumn{2}{c}{ With Wal-Mart } & \multicolumn{2}{c}{ No Unobserved } \\
& Time-aggregated & \multicolumn{2}{c}{ Entry Times } & \multicolumn{2}{c}{ Heterogeneity } \\
& Coeff. & S.E. & Coeff. & S.E. & Coeff. & S.E. \\
\hline Constant $\left(\theta_{1}^{\mathrm{f}}\right)$ & -13.074 & $(0.080)$ & -13.092 & $(0.080)$ & -12.698 & $(0.067)$ \\
Number of Chain stores $\left(\theta_{2}^{\mathrm{f}}\right)$ & -0.021 & $(0.003)$ & -0.021 & $(0.003)$ & -0.018 & $(0.003)$ \\
Number of Wal-Marts $\left(\theta_{2}^{\mathrm{f}}\right)$ & -0.041 & $(0.012)$ & -0.042 & $(0.012)$ & -0.054 & $(0.012)$ \\
Number of Fringe Stores $\left(\theta_{3}^{\mathrm{f}}\right)$ & 0.183 & $(0.008)$ & 0.183 & $(0.008)$ & 0.193 & $(0.008)$ \\
Number of Fringe Stores Squared / 100 (100 $\left.\times \theta_{4}^{\mathrm{f}}\right)$ & -0.349 & $(0.018)$ & -0.349 & $(0.019)$ & -0.369 & $(0.018)$ \\
Population $\left(\theta_{5}^{\mathrm{f}}\right)$ & 0.240 & $(0.021)$ & 0.248 & $(0.021)$ & 0.170 & $(0.021)$ \\
Unobserved State $\left(\theta_{6}^{\mathrm{f}}\right)$ & -2.530 & $(0.107)$ & -2.544 & $(0.107)$ & & \\
Unobserved State $\times$ Number of Fringe Stores $\left(\theta_{7}^{\mathrm{f}}\right)$ & 0.050 & $(0.006)$ & 0.051 & $(0.006)$ & & \\
& & & & & & \\
Entry Cost $\left(\eta_{0}^{\mathrm{f}}\right)$ & -5.034 & $(0.033)$ & -5.034 & $(0.033)$ & -5.030 & $(0.033)$ \\
Entry Cost $\times$ Unobserved State $\left(\eta_{1}^{\mathrm{f}}\right)$ & 1.186 & $(0.079)$ & 1.190 & $(0.079)$ & & \\
\hline
\end{tabular}


Table 5: Counterfactual Simulations of Market Structure in Year 2014 With and Without Wal-Mart

\begin{tabular}{|c|c|c|c|c|c|c|c|c|c|c|c|c|}
\hline & Markets & $\begin{array}{r}\text { Initial } \\
\text { Pop }\end{array}$ & $\begin{array}{l}\text { Chain } \\
\text { Firms }\end{array}$ & $\begin{array}{l}\text { Chain } \\
\text { Stores }\end{array}$ & $\begin{array}{l}\text { Fringe } \\
\text { Stores }\end{array}$ & $\begin{array}{r}\text { Wal-Mart } \\
\text { Stores }\end{array}$ & $\begin{array}{l}\text { Chain } \\
\text { Share }\end{array}$ & $\begin{array}{r}\text { Wal-Mart } \\
\text { Share }\end{array}$ & $\begin{array}{l}\text { Fringe } \\
\text { Share }\end{array}$ & $\mathrm{C} 1$ & $\mathrm{C} 3$ & HHI \\
\hline & \multicolumn{12}{|c|}{ With Wal-Mart } \\
\hline All Markets & 205 & 176153 & 2.41 & 9.17 & 11.98 & 2.42 & $39.9 \%$ & $10.8 \%$ & $49.4 \%$ & $25.4 \%$ & $48.1 \%$ & 0.22 \\
\hline Midwest & 58 & 175371 & 1.75 & 5.88 & 14.36 & 2.07 & $27.3 \%$ & $9.9 \%$ & $62.7 \%$ & $21.7 \%$ & $39.1 \%$ & 0.20 \\
\hline Northeast & 22 & 205180 & 2.18 & 8.48 & 14.32 & 2.58 & $35.2 \%$ & $10.7 \%$ & $54.1 \%$ & $24.0 \%$ & $45.3 \%$ & 0.21 \\
\hline South & 83 & 170856 & 2.78 & 11.72 & 9.63 & 2.85 & $49.1 \%$ & $12.1 \%$ & $38.7 \%$ & $29.1 \%$ & $55.8 \%$ & 0.24 \\
\hline \multirow[t]{2}{*}{ West } & 42 & 172494 & 2.71 & 9.02 & 12.11 & 1.96 & $41.3 \%$ & $9.3 \%$ & $49.4 \%$ & $23.8 \%$ & $46.6 \%$ & 0.20 \\
\hline & \multicolumn{12}{|c|}{ Absent Wal-Mart } \\
\hline All Markets & 205 & 176153 & 2.77 & 12.43 & 9.85 & 0.00 & $54.9 \%$ & $0.0 \%$ & $44.6 \%$ & $29.9 \%$ & $55.7 \%$ & 0.26 \\
\hline Midwest & 58 & 175371 & 2.13 & 8.41 & 11.81 & 0.00 & $42.0 \%$ & $0.0 \%$ & $58.0 \%$ & $27.6 \%$ & $47.5 \%$ & 0.25 \\
\hline Northeast & 22 & 205180 & 2.61 & 12.22 & 11.18 & 0.00 & $53.7 \%$ & $0.0 \%$ & $46.3 \%$ & $30.6 \%$ & $55.4 \%$ & 0.27 \\
\hline South & 83 & 170856 & 3.22 & 16.15 & 7.54 & 0.00 & $66.9 \%$ & $0.0 \%$ & $32.7 \%$ & $33.2 \%$ & $64.3 \%$ & 0.28 \\
\hline West & 42 & 172494 & 2.86 & 10.77 & 11.02 & 0.00 & $49.5 \%$ & $0.0 \%$ & $48.8 \%$ & $25.9 \%$ & $50.2 \%$ & 0.23 \\
\hline
\end{tabular}


Table 6: Counterfactual Simulations of Changes in Market Structure Due to Wal-Mart's Presence

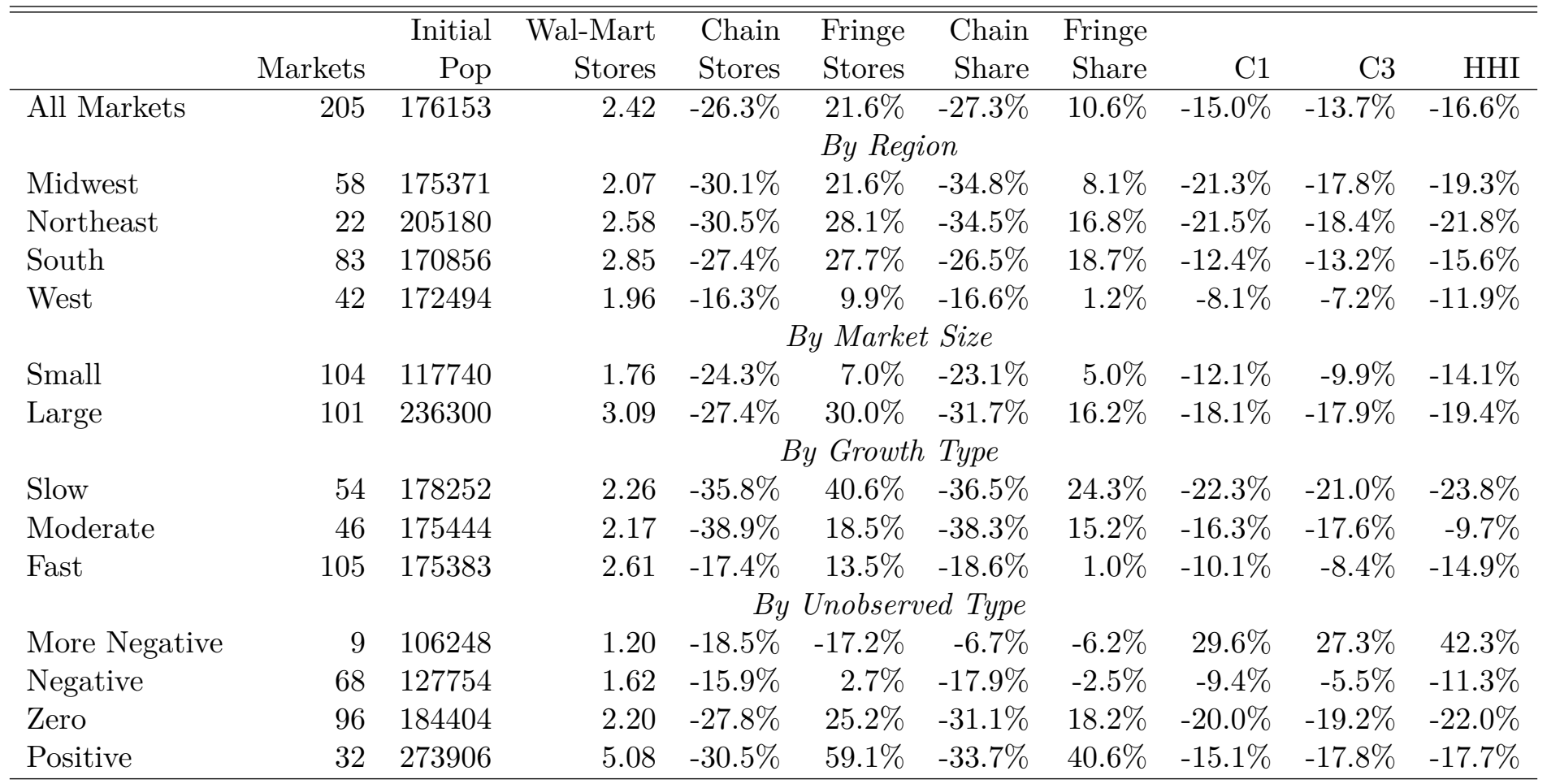


Table 7: Counterfactual Simulations of Changes in Market Structure Absent Unobserved Heterogeneity

\begin{tabular}{lrrrrrrrr}
\hline \hline & $\begin{array}{r}\text { Wal-Mart } \\
\text { Stores }\end{array}$ & $\begin{array}{r}\text { Chain } \\
\text { Stores }\end{array}$ & $\begin{array}{r}\text { Fringe } \\
\text { Stores }\end{array}$ & $\begin{array}{r}\text { Chain } \\
\text { Share }\end{array}$ & $\begin{array}{r}\text { Fringe } \\
\text { Share }\end{array}$ & C1 & C3 & HHI \\
\hline All Markets & 3.15 & $-33.2 \%$ & $7.3 \%$ & $-33.1 \%$ & $9.0 \%$ & $-12.9 \%$ & $-9.7 \%$ & $-7.0 \%$ \\
& & & \multicolumn{7}{c}{ By Region } \\
Midwest & 2.92 & $-38.4 \%$ & $9.7 \%$ & $-41.5 \%$ & $8.3 \%$ & $-17.2 \%$ & $-12.0 \%$ & $-6.6 \%$ \\
Northeast & 3.24 & $-35.8 \%$ & $7.0 \%$ & $-36.0 \%$ & $9.7 \%$ & $-14.6 \%$ & $-10.6 \%$ & $-7.5 \%$ \\
South & 3.28 & $-32.3 \%$ & $7.4 \%$ & $-29.8 \%$ & $11.7 \%$ & $-10.0 \%$ & $-8.5 \%$ & $-6.6 \%$ \\
West & 3.15 & $-28.6 \%$ & $3.4 \%$ & $-29.4 \%$ & $3.9 \%$ & $-12.4 \%$ & $-8.7 \%$ & $-7.6 \%$ \\
& & & \multicolumn{7}{c}{ By Market } & Size & & & \\
Large & 3.65 & $-30.5 \%$ & $7.3 \%$ & $-29.6 \%$ & $7.2 \%$ & $-12.2 \%$ & $-9.5 \%$ & $-7.8 \%$ \\
Small & 2.66 & $-37.5 \%$ & $7.1 \%$ & $-36.2 \%$ & $11.5 \%$ & $-13.9 \%$ & $-10.3 \%$ & $-6.8 \%$ \\
& & & & By Unobserved Type & & & \\
More Negative & 2.22 & $-42.9 \%$ & $15.6 \%$ & $-43.9 \%$ & $13.1 \%$ & $-16.6 \%$ & $-14.9 \%$ & $-2.9 \%$ \\
Negative & 2.71 & $-35.9 \%$ & $6.0 \%$ & $-36.9 \%$ & $8.5 \%$ & $-14.9 \%$ & $-9.8 \%$ & $-6.5 \%$ \\
Zero & 3.24 & $-33.7 \%$ & $6.7 \%$ & $-32.8 \%$ & $7.6 \%$ & $-12.2 \%$ & $-9.1 \%$ & $-6.2 \%$ \\
Positive & 4.07 & $-29.6 \%$ & $9.3 \%$ & $-24.4 \%$ & $13.7 \%$ & $-10.1 \%$ & $-10.1 \%$ & $-12.5 \%$ \\
\hline
\end{tabular}


Table 8: Temporal Evolution of Market Structure

\begin{tabular}{|c|c|c|c|c|c|c|c|c|c|}
\hline Year & Market Size & WM Stores & Chain Stores & Fringe Stores & Chain Share & Fringe Share & $\mathrm{C} 1$ & C3 & HHI \\
\hline 5 & Small & 0.58 & $-6.8 \%$ & $3.4 \%$ & $-7.9 \%$ & $-0.1 \%$ & $-5.7 \%$ & $-6.3 \%$ & $-9.9 \%$ \\
\hline 5 & Large & 0.87 & $-10.0 \%$ & $5.2 \%$ & $-9.9 \%$ & $2.9 \%$ & $-5.2 \%$ & $-7.5 \%$ & $-10.1 \%$ \\
\hline 10 & Small & 1.06 & $-13.7 \%$ & $5.4 \%$ & $-14.3 \%$ & $0.8 \%$ & $-9.3 \%$ & $-9.2 \%$ & $-14.3 \%$ \\
\hline 10 & Large & 1.76 & $-16.8 \%$ & $12.8 \%$ & $-18.3 \%$ & $6.6 \%$ & $-10.2 \%$ & $-12.0 \%$ & $-15.6 \%$ \\
\hline 15 & Small & 1.45 & $-19.5 \%$ & $6.5 \%$ & $-19.4 \%$ & $2.0 \%$ & $-11.5 \%$ & $-10.3 \%$ & $-15.4 \%$ \\
\hline 15 & Large & 2.52 & $-22.3 \%$ & $21.3 \%$ & $-25.6 \%$ & $11.1 \%$ & $-14.6 \%$ & $-15.2 \%$ & $-18.2 \%$ \\
\hline 20 & Small & 1.76 & $-24.3 \%$ & $7.0 \%$ & $-23.1 \%$ & $5.0 \%$ & $-12.1 \%$ & $-9.9 \%$ & $-14.1 \%$ \\
\hline 20 & Large & 3.09 & $-27.4 \%$ & $30.0 \%$ & $-31.7 \%$ & $16.2 \%$ & $-18.1 \%$ & $-17.9 \%$ & $-19.4 \%$ \\
\hline
\end{tabular}

